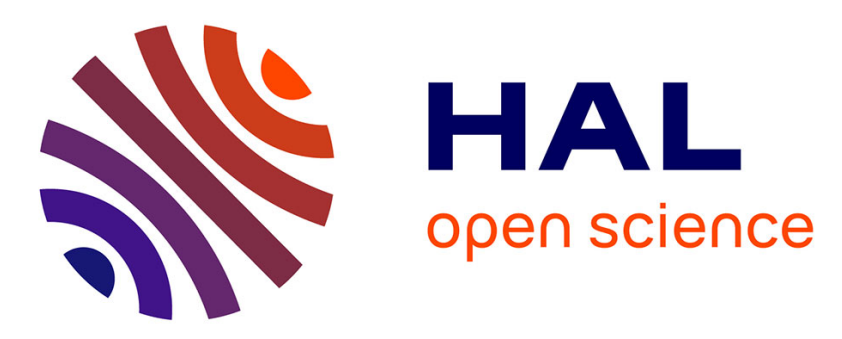

\title{
Peer-Assisted Individual Assessment in a multi-agent system
}

Wenjie Li, Francesca Bassi, Laura Galluccio, Michel Kieffer

\section{To cite this version:}

Wenjie Li, Francesca Bassi, Laura Galluccio, Michel Kieffer. Peer-Assisted Individual Assessment in a multi-agent system. Automatica, 2017, 83, pp.351 - 360. 10.1016/j.automatica.2017.06.031 . hal-01576605

\section{HAL Id: hal-01576605 \\ https://hal-centralesupelec.archives-ouvertes.fr/hal-01576605}

Submitted on 23 Aug 2017

HAL is a multi-disciplinary open access archive for the deposit and dissemination of scientific research documents, whether they are published or not. The documents may come from teaching and research institutions in France or abroad, or from public or private research centers.
L'archive ouverte pluridisciplinaire HAL, est destinée au dépôt et à la diffusion de documents scientifiques de niveau recherche, publiés ou non, émanant des établissements d'enseignement et de recherche français ou étrangers, des laboratoires publics ou privés. 


\title{
Peer-Assisted Individual Assessment in a Multi-Agent System
}

\author{
Wenjie Li ${ }^{\text {a }}$, Francesca Bassi ${ }^{\mathrm{b}, \mathrm{a}}$, Laura Galluccio ${ }^{\mathrm{c}}$, Michel Kieffer ${ }^{\mathrm{a}, \mathrm{d}}$ \\ ${ }^{a}$ L2S, CNRS, Supelec, Univ Paris-Sud, 3 rue Joliot-Curie, 91192 Gif-sur-Yvette, France \\ ${ }^{\mathrm{b}}$ ESME-Sudria, 38 rue Molière, 94200 Ivry-sur-Seine, France \\ ${ }^{\mathrm{c}}$ DIEEI, Università degli studi di Catania, viale Andrea Doria 6, Catania, Italy \\ ${ }^{\mathrm{d}}$ Institut Universitaire de France, 103 bld Saint-Michel, 75005 Paris
}

\begin{abstract}
Consider a multi-agent system where agents perform a given task with different levels of ability. Agents are initially not aware of how well they perform in comparison with their peers, and are willing to self-assess. This scenario is relevant, e.g., in wireless sensor networks, or in crowdsensing applications, where devices with embedded sensing capabilities collaboratively collect data to characterize the environment: the global performance is very sensitive to the measurement accuracy, and agents providing outliers should restrain to participate.

This paper presents a distributed algorithm enabling each agent to self-assess its own ability. The algorithm tracks the outcomes of a local comparison test performed by pairs of agents when they randomly meet, and able to gauge their relative level of ability. The dynamics of the proportions of agents with similar assessments are described using continuous-time state equations. The existence of an equilibrium is shown. Closed-form expressions for the various proportions of agents with similar assessments are provided at equilibrium. In simulations, a community of agents equipped with sensors, and trying to determine the performance of their equipment is considered. Simulation results show a good fitting with theoretical predictions.
\end{abstract}

Key words: Classification, Delay Tolerant Networks, Distributed estimation, Equilibrium, Multi-Agent Systems, Sensing

\section{Introduction}

Consider a community of agents collaborating to execute some task, e.g., sensing, detection, classification (see Ang et al., 2009; Luo et al., 2007; Shah et al., 2016). Agents are expected to have different levels of ability (LoAs) in carrying out atomic operations ${ }^{1}$. Initially, each one ignores how well it performs in comparison with the others, and is willing to assess its own LoA. This paper assumes that agents have only pairwise, sporadic interactions, as in delay tolerant networks (Khabbaz et al., 2012), or in networks where exchanges are performed via gossiping (Dimakis et al., 2010). This paper considers this distributed Peer-Assisted Individual Assessment

\footnotetext{
Email addresses: weli@12s.centralesupelec.fr (Wenjie Li), bassi@12s.centralesupelec.fr (Francesca Bassi), laura.galluccio@dieei.unict.it (Laura Galluccio), kieffer@12s. centralesupelec.fr (Michel Kieffer).

1 LOAs may be represented by positive integers or real numbers; here they are assumed discrete-valued.
}

(PAIA) problem, in which each agent of a community aims at learning its own LoA, in absence of any central ranking authority, only with the help of sporadic peerto-peer information exchanges.

algorithm to enable each agent to learn its own LoA in absence of a central ranking authority.

The PAIA algorithm is of interest in several scenarios. In a wireless sensor network (WSN) (Yick et al., 2008), for instance, devices with embedded sensors collaboratively collect data to characterize the surrounding environment. Agents who have only incomplete knowledge of the characteristics of the sensing noise (e.g., either biased or unbiased, as in (Chiuso et al., 2011)), may use the PAIA algorithm to estimate it. Similarly, in crowdsensing applications (Guo et al., 2015) data generated by personal mobile devices are collected in order to estimate some process. Since the reliability of the service depends on the accuracy of the measurements, the server prefers to pull data from the devices with the most accu- 
rate sensors. The problem of device selection is usually addressed by centralized reputation-based mechanisms, (see, e.g., Kantarci and Mouftah, 2014; Ren et al., 2015; Yu and Van der Schaar, 2012), where the devices apply via an auction system and the server selects on the basis of their established reputation level. Using the PAIA algorithm to assess their own accuracy, agents aware to be temporarily producing outliers may decide to restrain from an auction, to preserve their reputation at the central authority; agents aware that their accuracy is above the average may negotiate a better reward.

The PAIA problem can be viewed as a generalization of distributed faulty node detection (DFD). In DFD part of the nodes of a network are equipped with defective sensors producing measurement outliers (Mahapatro and Khilar, 2013; Zhang et al., 2010). Each node is willing to estimate the status of of its own sensor (good or defective) (Chen et al., 2006; Lee and Choi, 2008; Li et al., 2016a). DFD can thus be seen as a PAIA problem for two LoAs. This paper extends to PAIA the ideas introduced for DFD in (Li et al., 2016b) by considering more than two possible LoAs for each agent. In the proposed PAIA algorithm a pairwise interaction results in a local comparison test (LCT) able to gauge the relative strength of the participants. Each agent observes only the outcomes of the LCTs it has been involved in. Based on the proportion of interactions during which it has been deemed better, the agent is able to iteratively determine its own LoA. The algorithm parameters depend on the proportions of agents with the same LoA and on the probabilities of error of the LCT.

Ranking or classification by pairwise comparisons has been of interest for a long time. In this work we presuppose, as in classical models (Bradley and Terry, 1952; Luce, 1959; Thurstone, 1927), that an inherent partition of the agents according to their LoA exists, and that the outcomes of the comparisons are probabilistic. Unlike in parametric models, however, we limit the assumptions made on the matrix of the outcome probabilities. Besides classical win or lose pairwise comparisons, we account also for weaker LCTs, where the outcome only indicates whether the participants have comparable strength. Recent years are seeing renewed interest in ranking by pairwise comparisons (see, e.g. Heckel et al., 2016; Jamieson and Nowak, 2011; Negahban et al., 2012; Wauthier et al., 2013, and references therein). In these works a central authority observes the whole collection of outcomes, and usually directs the measurement process. In the PAIA problem, on the contrary, no agent centralizes all the data, and the agents cannot select the peers they interact with. Since distributed classification usually refers to agents cooperating to rate a set of objects, as in (Ang et al., 2009; Luo et al., 2007), whereas PAIA refers to agents rating themselves, PAIA can be defined as a distributed self-classification problem from pairwise comparisons.
The paper is organized as follows. Section 1.1 reviews additional related work. Section 2 introduces the system model and the LCT. Section 3 describes and analyzes the proposed PAIA algorithm. Its effectiveness is measured by the proportions of agents who assess their LoAs correctly. The analysis is performed by assuming a well-mixed population of agents, with intercontact delay following an exponential distribution (Galluccio et al., 2016; Hernandez-Orallo et al., 2015; Zhu et al., 2010).This communication model allows one to derive continuous-time state equations approximating the evolution in time of the proportions of agents with similar self-assessments. The existence of an equilibrium is shown in Section 4, and closed-form expressions for the proportions of agents with similar assessments at equilibrium are provided. The dependence of the correct decision rate and of the false decision rate on the characteristics of the LCT provides insights on the way the PAIA algorithm should be tuned to trade-off between them. Section 5 reports simulation results for a population of agents aiming to determine the LoA of their embedded sensors. The numerical results show and excellent match with the theoretical predictions. Finally, conclusions are drawn in Section 6.

\subsection{Related work}

In (Chiuso et al., 2011; Fagnani et al., 2014a,b) each node of a WSN estimates the value of some constant parameter from noisy measurements, jointly with the bias (Chiuso et al., 2011; Fagnani et al., 2014a) or the level of the variance (Fagnani et al., 2014b) of the noise. The bias or the variance determines the LoA of the agent. These works involve at least partially instances of the PAIA problem. In (Chiuso et al., 2011) the nodes belong to one of two classes, defined by the absence or presence of the bias. The algorithm involves a gossip consensus, robust against node mobility, and a distributed ranking of the agents (Fagnani and Zampieri, 2008) according to their measured value. The signal model is extended to vector measurements and to multiple values of the bias in (Fagnani et al., 2014a). Each node uses consensus (Huang and Manton, 2009) to achieve cooperation with the neighbors for the estimation of the constant parameter, while iteratively estimating its own local bias. Consensus algorithms are used also in (Fagnani et al., 2014b), where the two classes depend on the possible values of the noise variance. Notice that in (Chiuso et al., 2011; Fagnani et al., 2014a,b) the PAIA problem is solved by estimation and is thus bound to the considered signal model. The PAIA algorithm does not make assumptions on the nature of the measurements, but only presupposes a generic LCT, characterized by its probabilities of error. For example, the LCT may be based on taking a noisy measurement of some constant parameter as in (Chiuso et al., 2011), or on comparing the results of the same supervised image classification performed by two agents, or on a blitz-game when agents have to assess 
their level in a game.

When the number of possible LoAs equals the number of agents, the PAIA problem is equivalent to the distributed self-ranking problem addressed in a centralized way in (Heckel et al., 2016) and with a distributed approach as in (Fagnani and Zampieri, 2008).

\section{System Model and Local Comparison Test}

Consider a set $\mathcal{A}$ of $N_{\mathrm{A}}$ moving agents. Let $\theta_{i} \in \Theta=$ $\{1 \ldots K\}$ be the LoA of Agent $i . \mathcal{A}$ is partitioned into $K$ groups denoted $\mathcal{A}_{1} \ldots \mathcal{A}_{K}$, with $\mathcal{A}_{\theta}=\left\{i \in \mathcal{A}: \theta_{i}=\theta\right\}$. Denote $p_{\theta}$ the proportion of the agents belonging to $\mathcal{A}_{\theta}$. Without loss of generality, we assume that the groups are sorted in decreasing LoA: thus, the agents in $\mathcal{A}_{1}$ are the best-performing and those in $\mathcal{A}_{K}$ are the worstperforming. The following assumption is made:

- A1) $\theta_{i}(t)=\theta_{i}$, i.e., the LoA of Agent $i$ does not change during the experiment.

Agent $i$ is not aware of the actual value of $\theta_{i}$ but is willing to estimate it as fast as possible. To accomplish this it exploits information obtained during meetings with other agents. As in ( $\mathrm{Li}$ et al., 2016b), one considers the following assumptions:

- A2) only pairwise meetings are considered;

- A3) the agents form a well-mixed population, i.e. the probability that the next meeting of Agent $i$ will be with an agent belonging to $\mathcal{A}_{\theta}$ is proportional to $\left|\mathcal{A}_{\theta}\right|$;

- $A_{4}$ ) the time interval between two successive meetings of Agent $i$ with any other agent follows an exponential distribution with an inter-contact rate $\lambda$ (Galluccio et al., 2016; Zhu et al., 2010).

During a meeting two agents may engage in an interaction. Interactions take different forms depending on the application scenario, for example, the exchange of noisy measurements $m_{i}$ and $m_{j}$ of the same physical quantity when the agents are nodes of a WSN, or a blitz-game between humans, willing, e.g., to assess their playing level. It is assumed that a meeting does not necessarily entail interaction. Define $\alpha\left(\widehat{\theta}_{i}, \widehat{\theta}_{j}\right)$ as the probability of interaction of Agents $i$ and $j$ meeting at instant $t$. It is a function of the current estimates $\widehat{\theta}_{i}(t)$ and $\widehat{\theta}_{j}(t)$ of the agents. When Agents $i$ and $j$ meet, they exchange their estimated LoA. Agent $i$ will request an interaction with probability $\alpha\left(\widehat{\theta}_{i}, \widehat{\theta}_{j}\right)$ and Agent $j$ with a probability $\alpha\left(\widehat{\theta}_{j}, \widehat{\theta}_{i}\right)$. Interaction occurs when at least one of the agents requests it. Only the agent requesting an interaction acquires the resulting information.

The LCT is a procedure that Agent $i$ performs on the data exchanged during an interaction with Agent $j$. It allows Agent $i$ to gather some knowledge on its own LoA in comparison to the LoA of Agent $j$, but is unable to determine the absolute LoA of Agent $i$. The output $y_{i, j}$ of the LCT performed by Agent $i$ is binary, and yields $y_{i, j}=1$ when it is likely that Agent $i$ is not worse than Agent $j$, or when they have a similar LoA, depending on the application. The LCT may provide erroneous conclusions and is characterized by its statistical properties

$$
q\left(\theta_{i}, \theta_{j}\right)=\mathbb{P}\left\{Y_{i, j}=1 \mid \theta_{i}, \theta_{j}\right\}
$$

where $\mathbb{P}(\mathcal{E})$ denotes the probability of the event $\mathcal{E}$. One has not necessarily $q\left(\theta_{i}, \theta_{j}\right)=1-q\left(\theta_{j}, \theta_{i}\right)$. One considers the following assumptions on the LCT probabilities:

- A5) $\theta_{i} \leqslant \theta_{j}$ implies $q\left(\theta_{i}, 1\right) \geqslant q\left(\theta_{j}, 1\right)$;

- $A 6)$ for all $\left(\theta_{i}, \theta_{j}\right) \in \Theta^{2}$, one has $q\left(\theta_{i}, \theta_{j}\right)>0$.

A5) appears reasonable, since 1 is the highest LoA, and an agent with a very low LoA has less probability to conclude positively a LCT with the best-performing agents than an agent with intermediate expertise. A6) implies that even if $\theta_{i}$ is the worst LoA, the LCT has a nonzero probability to conclude that an agent in $\mathcal{A}_{i}$ is better than an agent in $\mathcal{A}_{j}$. This probability can be made arbitrarily small, but it is required positive to show the existence of an equilibrium of the dynamics describing the evolution of the proportions of agents with a given assessment, as discussed in Section 4.

An example of pairwise interaction and LCT is provided in Section 5 in the context of WSNs.

\section{PAIA Algorithm}

In a community of mobile agents able to interact and perform LCTs satisfying the assumptions in Section 2, a PAIA algorithm is the mechanism that enables each agent to estimate its own LoA. In the proposed algorithm, each agent manages two counters $c_{\mathrm{t}, i}(t)$ and $c_{\mathrm{b}, i}(t)$ initialized at 0 at $t=0$. The number of LCTs performed by Agent $i$ following an interaction it has requested is stored in $c_{\mathrm{t}, i}(t)$. The number of tests concluding that Agent $i$ is not worse than the agent met is stored in $c_{\mathrm{b}, i}(t)$. As indicated in Section 2, an agent involved in an interaction that it has not requested does not update $c_{\mathrm{t}, i}(t)$ and $c_{\mathrm{b}, i}(t)$. If the agents are randomly spread and move (Assumption $A 3$ ), the ratio $c_{\mathrm{b}, i}(t) / c_{\mathrm{t}, i}(t)$ will only depend on the proportions of agents in each group, on the interaction probabilities, and on the statistical properties of the LCT.

Intuitively, an agent with LoA $\theta_{i}$ is likely to have a larger ratio $c_{\mathrm{b}, i}(t) / c_{\mathrm{t}, i}(t)$ than an agent with $\operatorname{LoA} \theta_{j}^{\prime}$ when $\theta_{i}<$ $\theta_{j}^{\prime}$. One may thus introduce a partition of the interval $[0,1]$ into $K$ decision intervals $\left[\nu_{k}, \nu_{k-1}\right)$ with $\nu_{0}=1$ and 
$\nu_{K}=0$ and consider the decision rule

$$
\widehat{\theta}_{i}(t)=k \text { if } c_{\mathrm{b}, i}(t) / c_{\mathrm{t}, i}(t) \in\left[\nu_{k}, \nu_{k-1}\right), k=1 \ldots K
$$

The aim of this work is to determine conditions on $p_{1}, \ldots, p_{K}$ (proportion of agents in each group), and $q\left(\theta_{i}, \theta_{j}\right)$ (probability that the outcome of the LCT performed by $i$ is one) to show that the decision rule (2) leads to a satisfying assessment of the LoA of the agents, for all $k=1 \ldots K$.

\subsection{Practical PAIA Algorithm}

Let $\mathbf{x}_{i}(t)=\left(\theta_{i}, c_{\mathrm{t}, i}(t), c_{\mathrm{b}, i}(t)\right)$ represent the state of each Agent $i$. If all the LCT outcomes obtained in the past are considered, then $c_{\mathrm{t}, i}(t)$ can go unbounded, resulting in an infinite number of possible values for $\mathbf{x}_{i}(t)$. The global behavior of the algorithm is in this case difficult to analyze. To limit the number of possible states, one considers the evolution of $c_{\mathrm{t}, i}(t)$ and $c_{\mathrm{b}, i}(t)$ over a sliding variable-length time interval containing the time instants of the last $M$ meetings during which Agent $i$ has performed a LCT. Algorithm 1 summarizes the proposed PAIA algorithm for an arbitrary reference Agent $i$.

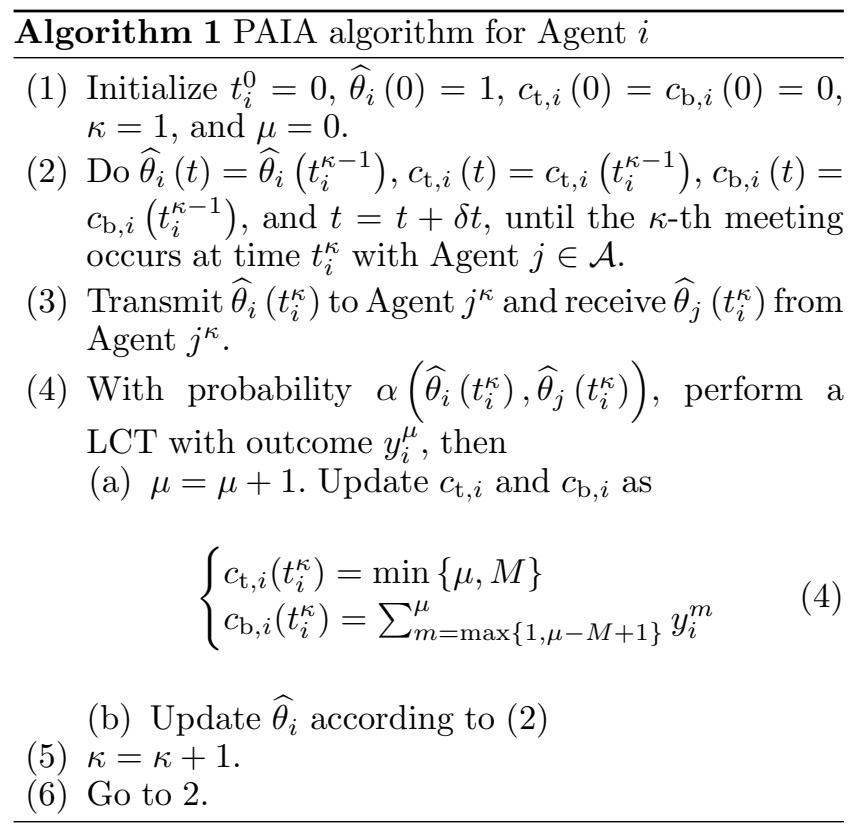

The behavior of the algorithm in accordance with the chosen performance criteria depends on the appropriate tuning of the thresholds $\nu_{k}$, as detailed in Section 4.3. These in general depend on the proportions $p_{\theta}$ of agents in each group, on the probabilities of interaction and on the statistical characteristics of the LCT. We assume this a priori information to be known at least to some agent management unit, responsible of setting up the network and of the diffusion of the values of the thresholds, during a short initialization phase.
The values of $\alpha\left(\widehat{\theta}_{i}, \widehat{\theta}_{j}\right)$ can be considered as design parameters that can be adjusted to optimize the performance of the PAIA algorithm. Since interaction is assumed to be associated to some cost for the agents, e.g., energy, in case of battery-powered devices, or time, in case of human players, they can also be tuned in order to restrain the total number of interactions per agent. Intuitively, keeping $\alpha\left(\widehat{\theta}_{i}, \widehat{\theta}_{j}\right)$ high if $\widehat{\theta}_{i}$ and $\widehat{\theta}_{j}$ are close and the outcome of the LCT is very uncertain, and keeping it low if $\widehat{\theta}_{i}$ and $\widehat{\theta}_{j}$ are far apart and the outcome of the LCT is easily foreseeable, restrains the number of interactions, while circulating the most relevant information.

\subsection{Macroscopic evolution}

At time $t$, among the agents with LoA $\theta$, let $X_{\theta}^{\tau, \beta}(t)$ be the proportion of agents in state $\mathbf{x}_{i}(t)=(\theta, \tau, \beta)$, i.e., with $c_{\mathrm{t}, i}(t)=\tau, c_{\mathrm{b}, i}(t)=\beta$, and $0 \leqslant \beta \leqslant \tau \leqslant M$. The evolution of the state of Agent $i$ with $\operatorname{LoA} \theta_{i}$, follows a Markov model with state transition diagram similar to the one shown in Figure 1 for $M=4$. There are $K$ parallel chains conditioned by the value of $\theta \in \Theta$. This diagram is similar to that considered in ( $\mathrm{Li}$ et al., 2016b). With the initial conditions $X_{\theta}^{0,0}(0)=1$ and $X_{\theta}^{\tau, \beta}(0)=0, \forall \tau, \beta \neq 0$, the evolution of expected value of the various proportions $X_{\theta}^{\tau, \beta}(t)$ of agents in the corresponding states, with $\theta=1 \ldots K$ and $0<\beta \leqslant \tau<M$, are described by (3) at the top of the page, see also ( $\mathrm{Li}$ et al., 2016b). In $(3), \pi_{\theta}^{\delta_{\mathrm{t}}, \delta_{\mathrm{b}}}$ denotes the transition probability from State $(\theta, \tau, \beta)$ to State $\left(\theta, \tau+\delta_{\mathrm{t}}, \beta+\delta_{\mathrm{b}}\right)$. Although the state equations (3) are the same as those in ( $\mathrm{Li}$ et al., 2016b), $\pi_{\theta}^{\delta_{\mathrm{t}}, \delta_{\mathrm{b}}}$ differs due to the increased number of groups and to the interaction probability $\alpha$.

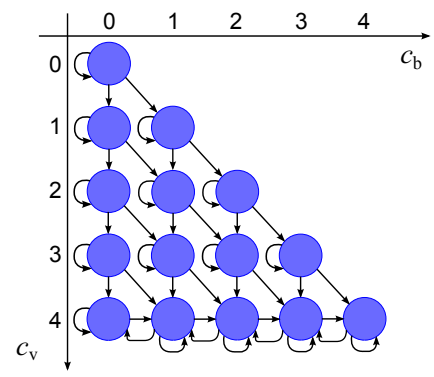

Fig. 1. Example of Markov model for the evolution of the state of a agent when $M=4$.

Note that $\pi_{\theta}^{\delta_{\mathrm{t}}, \delta_{\mathrm{b}}}$ depends on the current state $(\theta, \tau, \beta)$ of some reference agent, but also on the current proportion of agents with estimated LoA $\widehat{\theta}(t)$. One has first to evaluate the probability that some agent with state $(\theta, \tau, \beta)$, i.e., estimated LoA $\widehat{\theta}(\tau, \beta)$ performs a LCT during a meeting with a random agent, which index is described by the random variable $J$. This probability may be eval- 


$$
\left\{\begin{aligned}
& \frac{d X_{\theta}^{0,0}}{d t} \stackrel{(a)}{=}-\lambda X_{\theta}^{0,0} \cdot\left(\pi_{\theta}^{1,0}(0,0)+\pi_{\theta}^{1,1}(0,0)\right) \\
& \frac{d X_{\theta}^{\tau, 0}}{d t} \stackrel{(b)}{=} \lambda\left(-X_{\theta}^{\tau, 0} \cdot\left(\pi_{\theta}^{1,0}(\tau, 0)+\pi_{\theta}^{1,1}(\tau, 0)\right)+X_{\theta}^{\tau-1,0} \cdot \pi_{\theta}^{1,0}(\tau-1,0)\right) \\
& \frac{d X_{\theta}^{\tau, \tau}}{d t} \stackrel{(c)}{=} \lambda\left(-X_{\theta}^{\tau, \tau} \cdot\left(\pi_{\theta}^{1,0}(\tau, \tau)+\pi_{\theta}^{1,1}(\tau, \tau)\right)+X_{\theta}^{\tau-1, \tau-1} \cdot \pi_{\theta}^{1,1}(\tau-1, \tau-1)\right) \\
& \frac{d X_{\theta}^{M, 0}}{d t} \stackrel{(d)}{=} \lambda\left(-X_{\theta}^{M, 0} \cdot \pi_{\theta}^{0,1}(M, 0)+X_{\theta}^{M-1,0} \cdot \pi_{\theta}^{1,0}(M-1,0)+X_{\theta}^{M, 1} \cdot \pi_{\theta}^{0,-1}(M, 1)\right) \\
& \frac{d X_{\theta}^{M, M}}{d t} \stackrel{(e)}{=} \lambda\left(-X_{\theta}^{M, M} \cdot \pi_{\theta}^{0,-1}(M, M)+X_{\theta}^{M, M-1} \cdot \pi_{\theta}^{0,1}(M, M-1)+X_{\theta}^{M-1, M-1} \cdot \pi_{\theta}^{1,1}(M-1, M-1)\right) \\
& \frac{d X_{\theta}^{\tau, \beta}}{d t} \stackrel{(f)}{=} \lambda\left(-X_{\theta}^{\tau, \beta} \cdot\left(\pi_{\theta}^{1,0}(\tau, \beta)+\pi_{\theta}^{1,1}(\tau, \beta)\right)+X_{\theta}^{\tau-1, \beta} \cdot \pi_{\theta}^{1,0}(\tau-1, \beta)+X_{\theta}^{\tau-1, \beta-1} \cdot \pi_{\theta}^{1,1}(\tau-1, \beta-1)\right) \\
& \frac{d X_{\theta}^{M, \beta}}{d t} \stackrel{(g)}{=} \lambda\left(-X_{\theta}^{M, \beta} \cdot\left(\pi_{\theta}^{0,1}(M, \beta)+\pi_{\theta}^{0,-1}(M, \beta)\right)+X_{\theta}^{M-1, \beta-1} \cdot \pi_{\theta}^{1,1}(M-1, \beta-1)\right. \\
&\left.\quad+X_{\theta}^{M-1, \beta} \cdot \pi_{\theta}^{1,0}(M-1, \beta)+X_{\theta}^{M, \beta-1} \cdot \pi_{\theta}^{0,1}(M, \beta-1)+X_{\theta}^{M, \beta+1} \cdot \pi_{\theta}^{0,-1}(M, \beta+1)\right)
\end{aligned}\right.
$$

uated as $\gamma(\tau, \beta)=\mathbb{E}\left(\alpha\left(\widehat{\theta}(\tau, \beta), \widehat{\theta}_{J}(t)\right)\right)$, where $\mathbb{E}(\cdot)$ denotes the expectation, taken over $\widehat{\theta}_{J}(t)$. Then

$$
\begin{aligned}
& \gamma(\tau, \beta)=\sum_{k_{1}, k_{2} \in \Theta} \alpha\left(\widehat{\theta}(\tau, \beta), k_{2}\right) \mathbb{P}\left\{\widehat{\theta}_{J}(t)=k_{2}, \theta_{J}=k_{1}\right\} \\
& =\sum_{k_{1}, k_{2} \in \Theta} \alpha\left(\widehat{\theta}(\tau, \beta), k_{2}\right) \mathbb{P}\left\{\widehat{\theta}_{J}(t)=k_{2} \mid \theta_{J}=k_{1}\right\} \mathbb{P}\left\{\theta_{J}=k_{1}\right\} \\
& =\sum_{k_{1}, k_{2} \in \Theta} \alpha\left(\widehat{\theta}(\tau, \beta), k_{2}\right) p_{k_{1}} p^{k_{1} k_{2}}(t),
\end{aligned}
$$

where $p^{k_{1} k_{2}}(t)=\mathbb{P}\left\{\widehat{\theta}_{J}(t)=k_{2} \mid \theta_{J}=k_{1}\right\}$ is the proportion of agents with LoA $k_{1}$ believing their LoA is $k_{2}$. Using (2), one deduces that

$$
\begin{aligned}
& p^{k_{1} k_{2}}(t)=\mathbb{P}\left\{\widehat{\theta}_{J}(t)=k_{2} \mid \theta_{J}=k_{1}\right\} \\
& = \begin{cases}X_{k_{1}}^{0,0}(t)+\sum_{\tau, \beta, \beta / \tau \in\left[\nu_{k_{2}}, \nu_{k_{2}-1}\right)} X_{k_{1}}^{\tau, \beta}(t), & \text { if } k_{2}=1, \\
\sum_{\tau, \beta, \beta / \tau \in\left[\nu_{k_{2}}, \nu_{k_{2}-1}\right)} X_{k_{1}}^{\tau, \beta}(t), & \text { else. }\end{cases}
\end{aligned}
$$

For each agent, two phases have to be considered in Algorithm 1 , depending on the value of $c_{\mathrm{t}, i}(t)$. In the transient regime, for states with $c_{\mathrm{t}, i}(t)=\tau<M$, one has $\left(\delta_{\mathrm{t}}, \delta_{\mathrm{b}}\right) \in\{(0,0),(1,0),(1,1)\}$, since $\tau$ may either increase or remain constant and $\delta_{\mathrm{b}} \leqslant \delta_{\mathrm{t}}$. The only possibility leading to $\delta_{\mathrm{t}}=0$ is that Agent $i$, once it has met a random Agent $J$, decides not to interact. Then

$$
\pi_{\theta}^{0,0}(t, \tau, \beta)=1-\gamma(\tau, \beta)
$$

A state transition occurs with $\left(\delta_{\mathrm{t}}, \delta_{\mathrm{b}}\right)=(1,1)$ when, once Agent $i$ has met Agent $J$, they continue interacting and the LCT yields $y_{i, J}(t)=1$. Since $\alpha$ only depends on the estimates of the LoA, these two events can be assumed as independent and one has to consider all possible values taken by $\widehat{\theta}_{J}(t)$ to get

$$
\begin{aligned}
& \pi_{\theta}^{1,1}(t, \tau, \beta) \\
& =\sum_{k_{2} \in \Theta} \alpha\left(\widehat{\theta}(\tau, \beta), \widehat{\theta}_{J}(t)=k_{2}\right) \mathbb{P}\left\{Y_{i}=1, \widehat{\theta}_{J}(t)=k_{2} \mid \theta_{i}=\theta\right\} \\
& =\sum_{k_{1}, k_{2} \in \Theta} \alpha\left(\widehat{\theta}(\tau, \beta), k_{2}\right) \mathbb{P}\left\{Y_{i}=1 \mid \theta_{i}=\theta, \theta_{J}=k_{1}\right\} \\
& =\sum_{k_{1}, k_{2} \in \Theta} \alpha\left(\widehat{\theta}(\tau, \beta), k_{2}\right) p_{k_{1}} p^{k_{1} k_{2}}(t) q\left(\theta, k_{1}\right) .
\end{aligned}
$$

Then, $\pi_{\theta}^{1,0}(t, \tau, \beta)$ is obtained similarly:

$$
\begin{aligned}
& \pi_{\theta}^{1,0}(t, \tau, \beta) \\
& =\sum_{k_{1}, k_{2} \in \Theta} \alpha\left(\widehat{\theta}(\tau, \beta), k_{2}\right) p_{k_{1}} p^{k_{1} k_{2}}(t)\left(1-q\left(\theta, k_{1}\right)\right) .
\end{aligned}
$$

In the permanent regime, $c_{\mathrm{t}, i}(t)=M$ and remains constant, thus $\delta_{\mathrm{t}}=0$. In Algorithm 1, $\mu$ is the number of LCTs performed by Agent $i$ till time $t$. When $\mu \geqslant M$, only the last $M$ LCT outcomes are considered. To determine the value taken by $\delta_{\mathrm{b}} \in\{-1,0,1\}$ after the $\mu$-th LODT, consider an arbitrary $y \in\{0,1\}$ and the random event $\mathcal{E}_{y}(t)=\left\{Y_{i}^{\mu-M}=y \mid \sum_{m=\mu-M}^{\mu-1} Y_{i}^{m}=\beta\right\}$ which corresponds to a situation where one knows that $\beta$ LCTs yield 1 among the last $M$ tests and $Y_{i}^{\mu-M}=y$ will be ignored once the new LCT outcome is available. $\mathbb{P}\left\{\mathcal{E}_{1}(t)\right\}$ is relatively complex to evaluate, since $\mathbb{P}\left\{Y_{i}^{n}=y\right\}$ depends on the actual LoA of the encountered agent. In what follows, we assume that LCT outcomes with $Y_{i}^{m}=$ $y$ are independently distributed over the time horizon corresponding to $m=\mu-M \ldots \mu-1$. One obtains then $\mathbb{P}\left\{\mathcal{E}_{1}(t)\right\}=\beta / M$ and $\mathbb{P}\left\{\mathcal{E}_{0}(t)\right\}=1-\beta / M$.

Assume that the $(\mu-M)$-th LCT performed by Agent $i$ occurred at time $\tilde{t}$, then $y_{i}^{\mu-M}$ can also be denoted as $y_{i}(\tilde{t})$ and the transition related to $c_{\mathrm{b}, i}$ is such that $\delta_{\mathrm{b}}=$ $y_{i}(t)-y_{i}(\tilde{t}) \in\{-1,0,1\}$. To have $\left(\delta_{\mathrm{t}}, \delta_{\mathrm{b}}\right)=(0,1)$, three independent events have to occur: 1$)$ interaction has to 
continue once Agent $J$ has been met;2) $\left.y_{i, J}(t)=1 ; 3\right)$ $y_{i}(\tilde{t})=0$, i.e., $\mathcal{E}_{0}(t)$ occurred. The transition probability is then deduced using derivations similar to (8):

$$
\begin{array}{r}
\pi_{\theta}^{0,1}(t, M, \beta)=\frac{M-\beta}{M} \sum_{k_{1}, k_{2} \in \Theta} \alpha\left(\widehat{\theta}(M, \beta), k_{2}\right) \\
\cdot p_{k_{1}} p^{k_{1} k_{2}}(t) q\left(\theta, k_{1}\right) .
\end{array}
$$

Consider now $\left(\delta_{\mathrm{t}}, \delta_{\mathrm{b}}\right)=(0,-1)$. Using a similar approach one deduces

$$
\begin{aligned}
\pi_{\theta}^{0,-1}(t, M, \beta)=\frac{\beta}{M} \sum_{k_{1}, k_{2} \in \Theta} \alpha\left(\widehat{\theta}(M, \beta), k_{2}\right) \\
\cdot p_{k_{1}} p^{k_{1} k_{2}}(t)\left(1-q\left(\theta, k_{1}\right)\right) .
\end{aligned}
$$

Applying (10-11), $\pi_{\theta}^{0,0}(t, M, \beta)$ can be obtained from $\pi_{\theta}^{0,0}(t, M, \beta)=1-\pi_{\theta}^{0,1}(t, M, \beta)-\pi_{\theta}^{0,-1}(t, M, \beta)$.

\section{Analysis of Equilibrium}

In this section, the asymptotic behavior of the state equations (3) is characterized. Let $\bar{X}_{\theta}^{\tau, \beta}$ be the value at equilibrium of $X_{\theta}^{\tau, \beta}$. The proportion of agents with LoA $\theta$ estimating their LoA as $\widehat{\theta}$ depends on the partition of the interval $[0,1]$ introduced in $(2)$ :

$$
\bar{p}^{\theta \widehat{\theta}}=\sum_{\tau=1}^{M} \sum_{\beta: \beta / \tau \in\left[\nu_{\hat{\theta}}, \nu_{\hat{\theta}-1}\right)} \bar{X}_{\theta}^{\tau, \beta} .
$$

This analysis for general $\alpha$ is challenging, one thus considers the following two special cases.

Case I, where a meeting always leads to an interaction,

$$
\alpha\left(k_{1}, k_{2}\right)=1, \quad \forall k_{1}, k_{2} .
$$

Case II, where an interaction is only performed when an Agent $i$ meets an Agent $j$ estimating its LoA as $\widehat{\theta}_{j}=1$,

$$
\alpha\left(k_{1}, k_{2}\right)= \begin{cases}1 & \text { if } k_{2}=1 \\ 0 & \text { else. }\end{cases}
$$

\subsection{Case I}

To lighten the notations, introduce

$$
s_{\theta}=\sum_{k \in \Theta} p_{k} q(\theta, k),
$$

then $\pi_{\theta}^{\delta_{t}, \delta_{b}}$ introduced in Section 3.2 may be rewritten as

$$
\begin{cases}\pi_{\theta}^{1,1}(t, \tau, \beta)=s_{\theta}, & \pi_{\theta}^{0,1}(t, M, \beta)=\frac{M-\beta}{M} s_{\theta}, \\ \pi_{\theta}^{1,0}(t, \tau, \beta)=1-s_{\theta}, & \pi_{\theta}^{0,-1}(t, M, \beta)=\frac{\beta}{M}\left(1-s_{\theta}\right) .\end{cases}
$$

The transition probabilities are now time-invariant.

Proposition 1 In Case I, the dynamic system (3) admits an equilibrium $\bar{X}_{\theta}^{\tau, \beta}$, for all $\theta \in \Theta$ and $\beta \leqslant \tau$, with

$$
\bar{X}_{\theta}^{\tau, \beta}= \begin{cases}0, & \forall \tau<M, \\
\left(\begin{array}{c}
M \\
\beta
\end{array}\right)\left(s_{\theta}\right)^{\beta}\left(1-s_{\theta}\right)^{M-\beta}, & \tau=M .\end{cases}
$$

Proof See (Li et al., 2017), Section 7.1.

With the expression of $\bar{X}_{\theta}^{\tau, \beta}$ introduced in Proposition 1, one gets the correct decision rate (CDR) of agents with LoA $\theta \in \Theta$,

$$
\bar{p}^{\theta \theta}=\sum_{\beta: \beta / M \in\left[\nu_{\theta}, \nu_{\theta-1}\right)}\left(\begin{array}{c}
M \\
\beta
\end{array}\right)\left(s_{\theta}\right)^{\beta}\left(1-s_{\theta}\right)^{M-\beta} .
$$

The next proposition introduces a sufficient condition on the decision thresholds to get $\bar{p}^{\theta \theta} \rightarrow 1$ as $M \rightarrow \infty$.

Proposition 2 If $s_{1}>s_{2}>\ldots>s_{K}$ and for all $\theta \in$ $\Theta \backslash\{K\}, \nu_{\theta}<s_{\theta}<\nu_{\theta-1}$, then for all $\theta \in \Theta$,

$$
\lim _{M \rightarrow \infty} \bar{p}^{\theta \theta}=1 \text {. }
$$

Proof See (Li et al., 2017), Section 7.2

\subsection{Case II}

To perform the analysis of the equilibrium, one investigates first the evolution of $X_{\theta}^{\tau, k}(t)$ when $\tau<M$ in the following proposition.

Proposition 3 For any $\beta \leqslant \tau<M, \lim _{t \rightarrow \infty} X_{\theta}^{\tau, \beta}(t)=0$.

One can prove Proposition 3 by a simple extension of the proof of Proposition 2 in (Li et al., 2016b), in which $\theta$ takes a binary value. As a consequence, the only possible value at equilibrium of $X_{\theta}^{\tau, \beta}(t)$ with $\tau<M$ is 0 . In what follows, the analysis focuses on the agents performing an estimate $\widehat{\theta}=1$. The analysis for the other estimated values is similar.

Let $\overline{\mathbf{p}}^{1}=\left(\bar{p}^{11}, \ldots, \bar{p}^{K 1}\right)$ and consider the functions

$$
h_{\theta}\left(\overline{\mathbf{p}}^{1}\right)=\frac{\sum_{k \in \Theta} p_{k} \bar{p}^{k 1} q(\theta, k)}{\sum_{k \in \Theta} p_{k} \bar{p}^{k 1}},
$$


$F_{\theta}\left(\overline{\mathbf{p}}^{1}\right)=\sum_{\beta=\left\lceil\nu_{1} M\right\rceil}^{M}\left(\begin{array}{c}M \\ \beta\end{array}\right)\left(h_{\theta}\left(\overline{\mathbf{p}}^{1}\right)\right)^{\beta}\left(1-h_{\theta}\left(\overline{\mathbf{p}}^{1}\right)\right)^{M-\beta}$,

and $\mathbf{F}\left(\overline{\mathbf{p}}^{1}\right)=\left(F_{1}\left(\overline{\mathbf{p}}^{1}\right) \ldots F_{K}\left(\overline{\mathbf{p}}^{1}\right)\right)$. The following proposition provides a non-linear equation that has to be satisfied by $\overline{\mathbf{p}}^{1}$. Once this equation is solved, one can easily deduce the various $\bar{X}_{\theta}^{M, \beta}$ at equilibrium.

Proposition 4 If the dynamic system (3) admits some equilibrium $\bar{X}_{\theta}^{\tau, \beta}$, then for any $\theta \in \Theta$ and $\beta \leqslant \tau$,

$\bar{X}_{\theta}^{\tau, \beta}=\left\{\begin{array}{l}0, \forall \tau<M, \\ \left(\begin{array}{c}M \\ \beta\end{array}\right)\left(h_{\theta}\left(\overline{\mathbf{p}}^{1}\right)\right)^{\beta}\left(1-h_{\theta}\left(\overline{\mathbf{p}}^{1}\right)\right)^{M-\beta}, \quad \tau=M,\end{array}\right.$

where $\overline{\mathbf{p}}^{1}$ is the solution of

$$
\overline{\mathbf{p}}^{1}=\mathbf{F}\left(\overline{\mathbf{p}}^{1}\right) .
$$

Proof See (Li et al., 2017), Section 7.3.

The existence of $\bar{X}_{\theta}^{M, \beta}$ mainly depends on whether (23) has a solution $\overline{\mathbf{p}}^{1}$. For that purpose, one shows the existence of an equilibrium in Proposition 5 using Brouwer's fixed-point theorem.

Proposition 5 For any $0=\nu_{K}<\cdots<\nu_{1}<\nu_{0}=1$, (23) always admits a solution, and the dynamical system (3) has an equilibrium.

Proof See (Li et al., 2017), Section 7.4.

Similar to Proposition 2, a sufficient condition to have $\lim _{M \rightarrow \infty} \bar{p}^{\theta \theta}=1$ is stated in the following proposition.

Proposition 6 If $q(1,1)>q(2,1) \ldots>q(K, 1)$ and

$$
\begin{aligned}
& \max _{\theta \in \Theta \backslash\{1\}, k \in \Theta} q(\theta, k)<\nu_{1}<q(1,1), \\
& q(\theta+1,1)<\nu_{\theta}<q(\theta, 1), \quad \forall \theta \in \Theta \backslash\{1\}, \\
& \text { then } \lim _{M \rightarrow \infty} \bar{p}^{\theta \theta}=1, \forall \theta \in \Theta .
\end{aligned}
$$

Proof See (Li et al., 2017), Section 7.5.

Explicit expressions for $\bar{p}^{\theta 1}$ are difficult to obtain from (23). Since $\bar{p}^{\theta 1}$ with $\theta \neq 1$ represents the proportions of agents that have wrongly estimated their group, the vector $\overline{\mathbf{p}}^{1}=\left(\bar{p}^{11}, \ldots, \bar{p}^{K 1}\right)$ should be close to $\widetilde{\mathbf{p}}^{1}=$ $\left(\widetilde{p}^{11}, \ldots, \widetilde{p}^{K 1}\right)$. One has $\lim _{\mathbf{p}^{1} \rightarrow \widetilde{\mathbf{p}}^{1}} h_{\theta}\left(\mathbf{p}^{1}\right)=q(\theta, 1)$. Assuming that at equilibrium, $h_{\theta}\left(\overline{\mathbf{p}}^{1}\right) \simeq q(\theta, 1)$, using (22), $\bar{X}_{\theta}^{M, \beta}$ can be approximated as

$$
\widetilde{X}_{\theta}^{M, \beta}=\left(\begin{array}{c}
M \\
\beta
\end{array}\right)(q(\theta, 1))^{\beta}(1-q(\theta, 1))^{M-\beta},
$$

and follows thus a binomial distribution.

\subsection{Choice of $\nu$}

Using $\bar{X}_{\theta}^{M, \beta}$ (in Case I) or $\widetilde{X}_{\theta}^{M, \beta}$ (in Case II), one is able to optimize the decision thresholds introduced in (2). The value of the $\nu_{\theta} \mathrm{s}$ may for example be adjusted to maximize the CDR under some cumulated false decision rate (FDR) constraint evaluated using (26), but alternative performance requirements may be considered. The following proposition provides the values of $\nu_{\theta} \mathrm{s}$ that maximize the sum of CDR, i.e., $\sum_{\theta \in \Theta} \bar{p}^{\theta \theta}$ or $\sum_{\theta \in \Theta} \widetilde{p}^{\theta \theta}$.

Proposition 7 In Case I, if $\forall \theta=1 \ldots(K-1)$,

$$
\nu_{\theta}=\frac{\log \left(\frac{1-s_{\theta}}{1-s_{\theta+1}}\right)}{\log \left(\frac{s_{\theta+1}}{s_{\theta}} \frac{1-s_{\theta}}{1-s_{\theta+1}}\right)}
$$

where $s_{\theta}$ is introduced in (15), then $\sum_{\theta \in \Theta} \bar{p}^{\theta \theta}$ takes its maximum value.

In Case II, if $\forall \theta=1 \ldots(K-1)$,

$$
\nu_{\theta}=\frac{\log \left(\frac{1-q(\theta, 1)}{1-q(\theta+1,1)}\right)}{\log \left(\frac{q(\theta+1,1)}{q(\theta, 1)} \frac{1-q(\theta, 1)}{1-q(\theta+1,1)}\right)},
$$

then $\sum_{\theta \in \Theta} \widetilde{p}^{\theta \theta}$ takes its maximum value.

Proof See (Li et al., 2017), Section 7.6.

One sees that the values at equilibrium (17) and the decision thresholds in Case I are functions of the values of $s_{\theta}$, themselves functions of the a priori proportions of agents in each group $p_{\theta}$ and of the characteristics of the LCT $q\left(\theta_{i}, \theta_{j}\right)$. In Case II, the approximate values at equilibrium (26) and the thresholds are only functions of the characteristics of the LCT.

Under the assumption that it exists an inherent partition of the agents according to their LoAs, it is reasonable to assume that the set of LoAs is available as a priori information, along with the statistical properties of the LCT $q\left(\theta_{i}, \theta_{j}\right)$. Depending on the application it may be difficult to know the proportions of agents $p_{\theta}$. In this case the designer may set $\alpha$ as in Case II, to make the optimum thresholds $\nu_{\theta}$ independent on $p_{\theta}$.

\section{Simulation results}

Consider a set $\mathcal{A}$ of $N_{\mathrm{A}}$ moving agents with very limited communication range, forming a DTN such that Hypotheses A1-A4 are satisfied. Each agent is equipped with a sensor providing noisy observations

$$
m_{i}(\boldsymbol{x}, t)=\phi(\boldsymbol{x}, t)+w_{i}(t), \quad \forall i \in \mathcal{A}
$$


of some scalar field $\phi(\boldsymbol{x}, t)$ at the location $\boldsymbol{x}$ and at time $t$. The components $w_{i}(t)$ of the measurement noise at time $t$ in (29) are described as realizations of independently distributed Gaussian variables $W_{i} \sim \mathcal{N}\left(e_{i}, \sigma^{2}\right)$, where $e_{i}$ is some constant, agent-specific bias. The bias terms $e_{i}, i \in \mathcal{A}$, are assumed to be realizations of iid zero-mean Laplacian random variables $E_{i}$ with parameter $\gamma$. The sensors with small $e_{i}$ provide better measurements. Introducing some constant parameter $\epsilon>0$, the set of agents is partitioned into $K$ groups defined as $\mathcal{A}_{\theta}=$ $\left\{i \in \mathcal{A}: \Lambda_{\theta-1} \leqslant\left|e_{i}\right|<\Lambda_{\theta}\right\}$, where $\Lambda_{\theta}=\epsilon \theta / \gamma, \forall \theta \in \Theta \backslash$ $\{K\}$ and $\Lambda_{\theta}=\infty$ when $\theta=K$. Therefore, the proportion of agents with LoA $\theta$ is $p_{\theta}=\exp (-\epsilon(\theta-1) / \gamma)-$ $\exp (-\epsilon \theta / \gamma)$. The LoA $\theta$ of an agent represents thus the group to which it belongs and provides an indication of the level of its bias. This problem is a generalization of that introduced in (Chiuso et al., 2011), where $\phi(\boldsymbol{x}, t)$ is assumed constant with location and time, and where the bias can only take discrete values.

The agents do not know their measurement bias and Algorithm 1 is used to allow each agent to estimate it. Two agents, when meeting at some location $\boldsymbol{x}$ and time $t$, perform two independent measurements of $\phi(\boldsymbol{x}, t)$. Then, both agents exchange the estimate of their respective $\theta$ to determine if they want to continue interacting. If this is the case, they exchange the measurements and run individually the LCT introduced in Section 5.1.

\section{$5.1 L C T$}

Consider a measurement $m$, some tolerance $\omega$, and the interval $[m]=[m-\omega, m+\omega]$ of width $2 \omega$ centered around $m$. Consider now Agents $i$ and $j$ meeting at time $t$ and exchanging the measurements $m_{i}$ and $m_{j}$ they performed at time $t$ and at the same location $\boldsymbol{x}$. The set estimate (Belforte et al., 1990) of $\phi(\boldsymbol{x}, t)$ obtained from $m_{i}$ and $m_{j}$ is defined as $\left[\widehat{\phi}\left(m_{i}, m_{j}\right)\right]=\left[m_{i}\right] \cap\left[m_{j}\right]$. If $\left[\widehat{\phi}\left(m_{i}, m_{j}\right)\right] \neq \emptyset$ it is likely that the biases $e_{i}$ and $e_{j}$ are close. Both agents can conclude that their sensors perform similarly. If $\left[\widehat{\phi}\left(m_{i}, m_{j}\right)\right]=\emptyset$, it is likely that $e_{i}$ and $e_{j}$ differ significantly more than $\omega$. In this case the agent is unable to determine whether it is carrying the best sensor. As a consequence, it cannot conclude that its sensor is not worse than that of the other agent. One thus obtains the following low-complexity LCT:

$$
y_{i, j}=y_{j, i}= \begin{cases}1, & \text { if }\left[\widehat{\phi}\left(m_{i}, m_{j}\right)\right] \neq \emptyset \\ 0, & \text { else }\end{cases}
$$

For any arbitrary pair of agents $i$ and $j$, with respective LoA $\theta$ and $\theta^{\prime}$, one is able to evaluate the probability $q\left(\theta, \theta^{\prime}\right)=\mathbb{P}\left\{Y_{i, j}=1 \mid i \in \mathcal{A}_{\theta}, j \in \mathcal{A}_{\theta^{\prime}}\right\}$ as a function of $\omega, \sigma$, and $\gamma$.
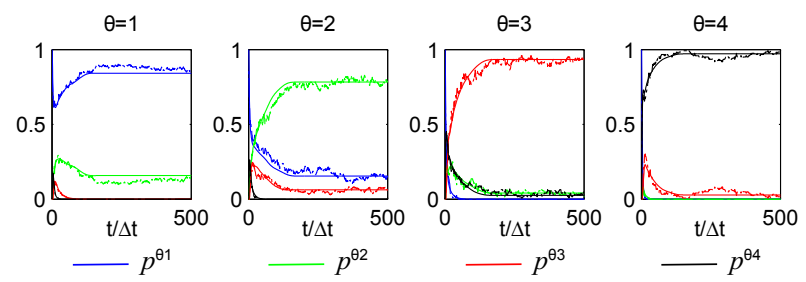

Fig. 2. Case I: Evolution of $p^{\theta \widehat{\theta}}(t)$, lines are for the theoretical values obtained solving (3), dots are simulation results using the random displacement model.

\subsection{Numerical verification of theoretical results}

This section presents first the solution of the state equation (3) describing the evolution of the proportion of nodes in various states. Algorithm 1 is analyzed first considering a random displacement of agents without constraint on their speed.

For the numerical example, one takes $K=4, \sigma^{2}=0.16$, and $\gamma=\epsilon=0.7$, resulting in

$$
p_{1}=0.503, p_{2}=0.250, p_{3}=0.124, p_{4}=0.123 \text {. }
$$

Moreover, taking $\omega=1.8$, one gets

$$
\mathbf{q}=\left(\begin{array}{llll}
0.95 & 0.66 & 0.22 & 0.01 \\
0.66 & 0.52 & 0.44 & 0.10 \\
0.22 & 0.44 & 0.50 & 0.27 \\
0.01 & 0.10 & 0.27 & 0.35
\end{array}\right)
$$

which satisfies Assumption A5. Besides, one considers $M=50$, and a sampling period $\Delta t$ during which the inter-contact probability is $\lambda \Delta t=0.33$. The decision thresholds are chosen using Proposition 7:

- In Case I, $\nu_{1}=0.61, \nu_{2}=0.42$, and $\nu_{3}=0.19$;

- In Case II, $\nu_{1}=0.84, \nu_{2}=0.43$, and $\nu_{3}=0.07$.

Figure 2 and 3 presents the evolution $p^{\theta \widehat{\theta}}(t)$ for $\theta, \widehat{\theta} \in$ $\Theta=\{1,2,3,4\}$, in Case I and in Case II respectively. One observes that the proportions of agents in each state converge. Moreover, in Case II, for any $\theta \in \Theta, p^{\theta \theta}$ is close to 1 for $t$ sufficient large, while $p^{\widehat{\theta} \widehat{\theta}}$ tends to 0 for any $\widehat{\theta} \neq \theta$. Nevertheless, in Case I, Algorithm 1 does not behave in a satisfying way: the values of $p^{11}$ and $p^{22}$ at the equilibrium are around 0.8 , which means that $20 \%$ of the nodes with LoA $\theta=1$ or $\theta=2$ do not correctly rate their LoA.

Consider now a set $\mathcal{A}$ of $N_{\mathrm{A}}=1000$ moving agents with initial positions uniformly distributed over a unit square. The agents randomly move within this square. Two agents communicate only at discrete time instants $k \Delta t, k=1,2 \ldots$ and when their distance is less than 

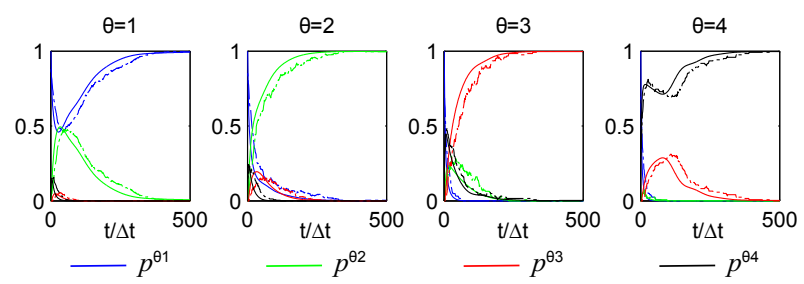

Fig. 3. Case II: Evolution of $p^{\theta \widehat{\theta}}(t)$, lines are for the theoretical values obtained solving (3), dots are simulation results using the random displacement model.

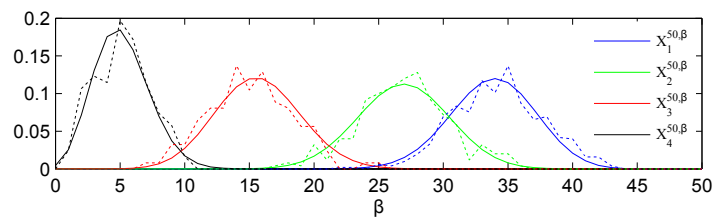

Fig. 4. Comparison of $\bar{X}_{\theta}^{50, \beta}$ at the equilibrium in Case I: lines are for the theoretical values obtained integrating (3) and dotted lines are for the moving agents simulation.

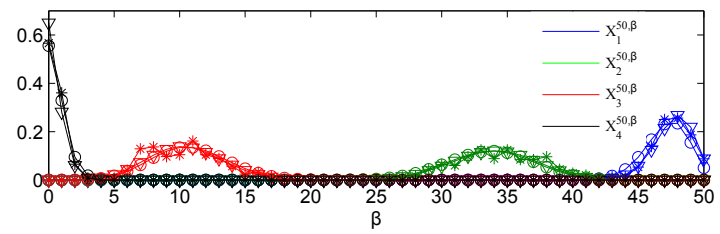

Fig. 5. Comparison of $\bar{X}_{\theta}^{50, \beta}$ at the equilibrium in Case II: circles are for the theoretical values obtained integrating (3), triangles are the approximations obtained from (26), and crosses are for the moving agents simulation.

$r_{0}$. One assumes that the displacement is fast enough so that the location of each agent at time $(k+1) \Delta t$ is independent of its location at time $k \Delta t$. Let $\mathcal{N}_{i}(k \Delta t)=$ $\left\{j \in \mathcal{A}: 0<r_{i, j}(k \Delta t) \leqslant r_{0}\right\}$, where $r_{i, j}(k \Delta t)$ is the distance between Agents $i$ and $j$ at time $k \Delta t$. Agent $i$ communicates with its neighbors if and only if the cardinal number $\left|\mathcal{N}_{i}(k \Delta t)\right|$ of $\mathcal{N}_{i}(k \Delta t)$ satisfies $\left|\mathcal{N}_{i}(k \Delta t)\right| \geqslant 1$. If $\left|\mathcal{N}_{i}(k \Delta t)\right|>1$, pairwise communications are considered. One may easily show that the average value of $\left|\mathcal{N}_{i}(k \Delta t)\right|$ is $\rho=\pi r_{0}^{2} N_{\mathrm{A}} \cdot\left|\mathcal{N}_{i}(k \Delta t)\right|$ approximately follows a Poisson distribution provided that $N_{\mathrm{A}}$ is large enough. The inter-contact probability at $k \Delta t$ is thus $\lambda \Delta t=\mathbb{P}\left\{\left|\mathcal{N}_{i}\right| \geqslant 1\right\}=1-\exp (-\rho)$.

In the following simulations, one considers $r_{0}=0.014$, so that $\rho \approx 0.6$ and $\lambda \Delta t \approx 0.33$. The evolutions of $p^{\hat{\theta}}(t)$ are also shown (dotted lines) in Figure 2 and 3 for Case I and Case II respectively. They are close to those predicted by the direct integration of the state equation (3).

Figure 5 further illustrates the good match between theory and simulation for the proportions of states $\bar{X}_{\theta}^{50, \beta}$ at equilibrium in Case I. In Case II, Figure 5 shows also a very good match between the simulation and the approximation of $\bar{X}_{\theta}^{50, \beta}$ obtained using (26).

\subsection{Simulation with real databases}

In this section, Algorithm 1 is simulated considering the intercontact time instants provided by two databases extracted from the MIT Reality Mining Project (Eagle and Pentland, 2006) and the Haggle Project (Scott et al., 2009), which have been considered in previous works, see, e.g., (Hui et al., 2011). In this work, we use the following databases:

- Reality, taken from (Eagle and Pentland, 2006), lasts more than 200 days and involves $N_{\mathrm{A}}=97$ agents with about 111 inter-contacts per day.

- Infocom05, taken from (Scott et al., 2009), lasts 3 days and involves $N_{\mathrm{A}}=41$ agents with approximately 312 inter-contacts every hour.

The inter-contact time instants were taken from (Orlinski, 2016), which are converted from the original databases (Eagle and Pentland, 2006; Scott et al., 2009).

For each database, 100 independent simulations are performed and results are averaged over these simulations. As in Section 5.2, $K=4$ groups are considered. The LoA of each node is randomly chosen according to (31). When an agent decides to continue interaction with an other agent, instead of performing a measurement and a test, a LCT is simulated with outcome randomly generated using (32).

\subsubsection{Case I}

At the top of Figure 6, for each time instant, a dot on the line corresponding to the index of an agent represents a contact with an other agent. The bottom of Figure 6 presents the evolution with time of the proportions $p_{e}^{\theta}=1-p^{\theta \theta}$ of nodes erroneously assessing their LoA for each $\theta$, considering $M=50$ and $M=200$. One also observes that the convergence speed of $p_{e}^{\theta}$ is highly related to the inter-contact rate (reflected by the density of points in the top sub-figures): variations are significant at beginning of working hours.

Figure 6 as well as the values at equilibrium of $\bar{X}_{\theta}^{M, \beta}$ reported in Figure 9 show some mismatch between theory and simulations for the agents with $\operatorname{LoA} \theta=1$ and $\theta=2$. Although there are many inter-contacts, some $X_{\theta}^{M, \beta} \mathrm{s}$ have not reached their theoretical values, especially for $\theta=1$ and $\theta=2$. This effect is more significant when $M$ is small, which is consistent with Proposition 2: the behavior of the PAIA algorithm improves when $M$ increases. After investigation of the trace, one observes that some agents have only few contacts with other agents. For example, 4 agents have less than 100 contacts with other agents. Moreover, the variety of agents met by some agents is limited. For example, 4 nodes have contacts with less than 10 different other nodes. This has a significant influence on the performance of the algorithm. 

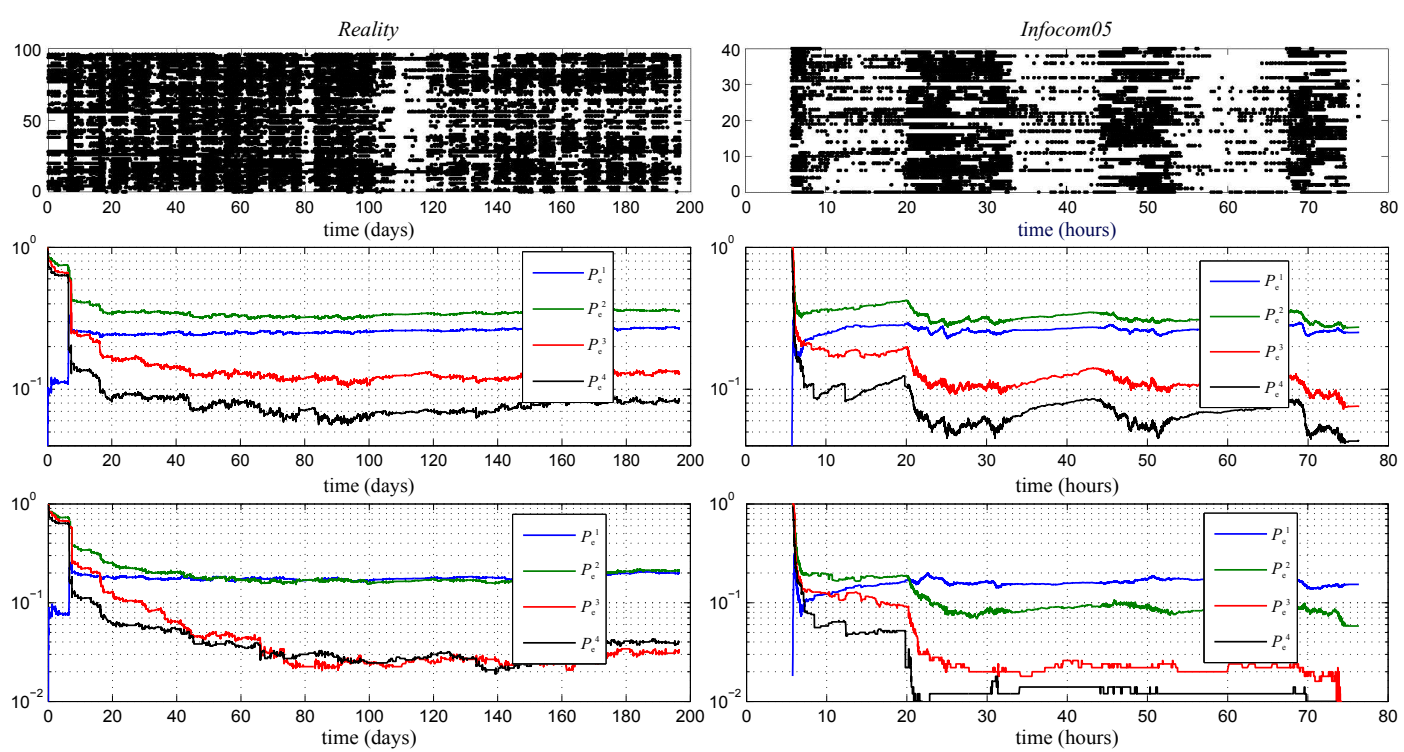

Fig. 6. Case I: Indexes of active nodes (having met another node) at different time (top) and evolution of $P_{e}^{\theta}=1-p^{\theta \theta}$ when $M=50$ (middle) and $M=200$ (bottom) obtained using the Reality database (left) and the Infocom05 database (right).
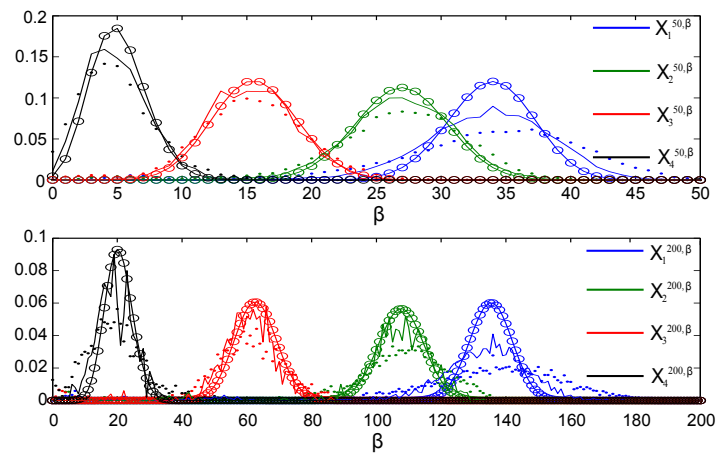

Fig. 7. Case I: Values of $\bar{X}_{\theta}^{50, \beta}$ (top) and of $\bar{X}_{\theta}^{200, \beta}$ (bottom) at the end of the simulation: circles are for the theoretical values at equilibrium obtained from (3), dots are simulation results using the Reality database, and plain lines are simulation results using the Infocom05 database.

\subsubsection{Case II}

Figure 8 shows the evolution of $p_{e}^{\theta}$ in Case II. For both databases, $p_{e}^{\theta}$ remains around $10^{-2}$ for all $\theta \in \Theta$ after a sufficient long time.

Figure 9 represents $\bar{X}_{\theta}^{M, \beta}$ obtained by using the databases Reality and Infocom05, and also by the approximation (9). In this case, there is an excellent match between the values at equilibrium predicted by theory and those obtained in simulation.

\subsubsection{Comparison}

Comparing the theoretical values of $\bar{X}_{\theta}^{50, \beta}$ at equilibrium for different values of $\theta \in \Theta$, one observes from Figures 7 and 9 that the different curves overlap more in Case I,

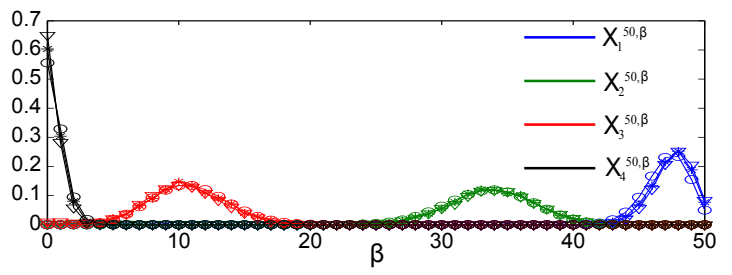

Fig. 9. Case II: Values of $\bar{X}_{\theta}^{50, \beta}$ at the end of simulation: circles are for the theoretical values obtained from (3), triangles are simulation results using the Reality database, and crosses are simulation results using the Infocom05 database.

especially those for $\theta=1$ (blue) and $\theta=2$ (green) and overlap much less in Case II. As a consequence, when considering some decision thresholds, better assessment quality will be obtained in Case II. This illustrates the importance of the probability of interaction $\alpha\left(\widehat{\theta}_{i}, \widehat{\theta}_{j}\right)$. In the considered application scenario, a better assessment is obtained when agents only perform LCT with agents believing their LoA is the best one.

\subsection{Comparison with state-of-the-art solution}

As discussed in Section 1.1, a few solutions for instances of the PAIA algorithm in the context of estimation in WSN exist in literature. The aim of this section is to compare the proposed PAIA algorithm with the solution proposed in (Fagnani et al., 2014a). The measurement model considered in (Fagnani et al., 2014a) can be captured by (29), by imposing that all the agents observe the same phenomenon independently on their location $\phi\left(\boldsymbol{x}_{i}, t\right)=\phi(t)$, and by imposing that the bias term $e_{i}$ of the measurement noise $W_{i}$ takes value from a finite alphabet $\mathcal{C}$, e.g., $\mathcal{C}=\{0,1,2\}$. The set of agents is then 

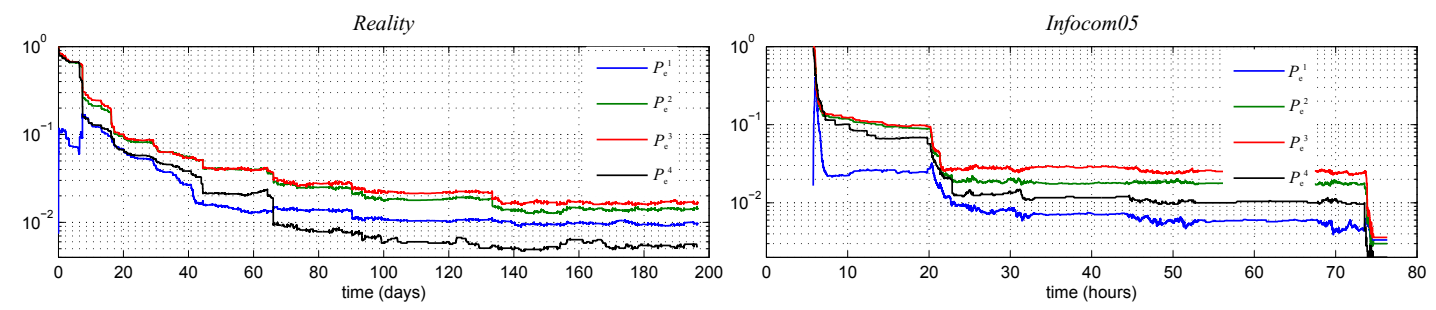

Fig. 8. Case II: Evolution of $P_{e}^{\theta}=1-p^{\theta \theta}$ obtained using the Reality database (left) and the Infocom05 database (right)

partitioned into $|\mathcal{C}|$ groups with respect to the their values of $e_{i}$. In (Fagnani et al., 2014a) each agent estimates $\phi$ and $e_{i}$ jointly using a Distributed Maximum Likelihood (DML) algorithm. Considering the network topology in this paper, the DML algorithm can be performed as follows: when Agent $i$ meets Agent $j$ at time $t$, it updates its estimation as

$$
\begin{aligned}
& \widehat{\phi}_{i}\left(t^{+}\right)=(1-\varsigma) \frac{\widehat{\phi}_{i}(t)+\widehat{\phi}_{j}(t)}{2}+\varsigma\left(m_{i}(t)-\widehat{e}_{i}(t)\right) \\
& \widehat{e}_{i}\left(t^{+}\right)=\arg \min _{e \in \mathcal{C}}\left(m_{i}(t)-\widehat{\phi}_{i}(t)-e\right)^{2}
\end{aligned}
$$

where $\varsigma \in(0,1)$ is a balance parameter.

As a comparison, one can use the proposed PAIA algorithm in order to estimate $e_{i}$ and simultaneously employ (33) to estimate $\phi$. Consider a simple numerical example where $\phi=1, \sigma=0.3$, and $\mathcal{C}=\{0,1\}$. Using the LCT introduced in Section 5.1 with $\omega=0.35$ one has

$$
\mathbf{q}=\left(\begin{array}{ll}
0.90 & 0.24 \\
0.24 & 0.90
\end{array}\right)
$$

The value of $\nu$ is set according to Proposition 7 . Since the entries of $\mathbf{q}$ in (35) violate the sufficient condition (24) in Proposition 6, the performance of the PAIA algorithm can be verified only numerically in Case II.

Consider the database Infocom05 with $n_{2}=20$ randomly chosen agents with $e=1$ and the other $n_{1}=21$ agents with $e=0$. Results are obtained as the average of 1000 independent Monte-Carlo simulations. Figure 10 shows the results for $\sigma=0.3$, where the classification error and the estimation error are obtained by $\sum\left|e_{i}-\widehat{e}_{i}\right| / N_{\mathrm{A}}$ and $\sum\left|\phi-\widehat{\phi}_{i}\right| / N_{\mathrm{A}}$ respectively. One observes that the proposed PAIA algorithm performs better than the reference method: both types of error decrease faster and are smaller.

\section{Conclusions}

This paper considered the problem of helping agents assessing their LoA at doing some task via the exchange of information with peers. Using a generic LCT involving,
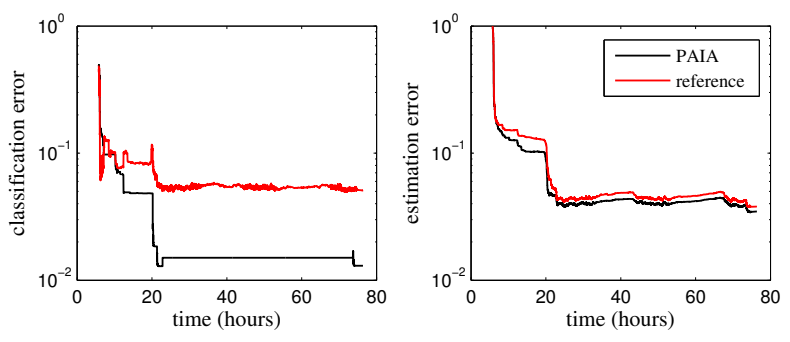

Fig. 10. Comparison of the reference algorithm proposed in (Fagnani et al., 2014a) with the PAIA algorithm.

e.g., data exchanged during meetings with other agents, each agent is able to estimate the proportion of agents it is better at doing the considered task. With that information, each agent may then determine to which group of agents with similar expertise it belongs to.

The behavior of the proposed algorithm is described using dynamical state equations approximating the evolution of the proportions of agents with similar beliefs in their LoA. Sufficient conditions for the existence of an equilibrium for these equations are established. The equilibrium is then characterized. This gives some insight in tuning the various parameters of the proposed algorithm.

The approach is illustrated with a crowdsensing scenario, where agents equipped with sensing devices of different performance, are willing to estimate the quality of their sensor. Simulation results show a good match with theory provided that the probabilities of interactions are such that the LCTs are performed only with the agents deeming their sensors are of the best quality.

Significant work remains to be done to analyze the behavior of the proposed algorithm with generic probabilities of interaction. The existence and uniqueness of the equilibrium has also to be shown in the general case. Nevertheless, the proposed approach may be useful to analyze other types of individual assessment problems.

\section{References}

Ang, H. H., Gopalkrishnan, V., Ng, W. K., Hoi, S., 2009. Communication-efficient classification in P2P networks. In: Proc. Joint Eur. Conf. on Mach. Learn. 
and Knowl. Disc. in Databases. Bled, Slovenia, pp. 83-98.

Belforte, G., Bona, B., Cerone, V., 1990. Parameter estimation algorithms for a set-membership description of uncertainty. Automatica 26 (5), 887-898.

Bradley, R. A., Terry, M. E., 1952. Rank analysis of incomplete block designs: I. The method of paired comparisons. Biometrika 39 (3/4), 324-345.

Chen, J., Kher, S., Somani, A., 2006. Distributed fault detection of wireless sensor networks. In: Proc. Workshop on Dependability Issues in Wireless Ad Hoc Networks and Sensor Networks. Los Angeles, CA, pp. 65 -72 .

Chiuso, A., Fagnani, F., Schenato, L., Zampieri, S., 2011. Gossip algorithms for simultaneous distributed estimation and classification in sensor networks. IEEE Jnl Sel. Top. Sig. Proc. 5 (4), 691-706.

Cover, T. M., Thomas, J. A., 2012. Elements of information theory. John Wiley \& Sons.

Dimakis, A. G., Kar, S., Moura, J. M. F., Rabbat, M. G., Scaglione, A., 2010. Gossip algorithms for distributed signal processing. Proc. of the IEEE 98 (11), 1847 1864.

Eagle, N., Pentland, A., 2006. Reality mining: sensing complex social systems. Personal and ubiquitous computing 10 (4), 255-268.

Fagnani, F., Fosson, S. M., Ravazzi, C., 2014a. Consensus-like algorithms for estimation of Gaussian mixtures over large scale networks. Math. Models and Methods in Applied Sciences 24 (02), 381-404.

Fagnani, F., Fosson, S. M., Ravazzi, C., 2014b. A distributed classification/estimation algorithm for sensor networks. SIAM Jnl on Cont. and Optim. 52 (1), 189-218.

Fagnani, F., Zampieri, S., 2008. Randomized consensus algorithms over large scale networks. IEEE Jnl on Sel. Areas in Com. 26 (4), 634-649.

Galluccio, L., Lorenzo, B., Glisic, S., 2016. Socialityaided new adaptive infection recovery schemes for multicast DTNs. IEEE Trans. Veh. Techn. 65 (5), 3360-3375.

Granas, A., Dugundji, J., 2013. Fixed point theory. Springer Science \& Business Media.

Guo, B., Wang, Z., Yu, Z., Wang, Y., Yen, N.Y. andHuang, R., Zhou, X., September 2015. Mobile crowd sensing and computing: the review of an emerginghuman-powered sensing paradigm. ACM Computing Surveys 48 (1).

Heckel, R., Shah, N. B., Ramchandran, K., Wainwright, M. J., 2016. Active ranking from pairwise comparisons and the futility of parametric assumptions. arXiv preprint arXiv:1606.08842.

Hernandez-Orallo, E., Serrat Olmos, M. D., Cano, J.-C., Calafate, C. T., Manzoni, P., 2015. Cocowa: A collaborative contact-based watchdog for detecting selfish nodes. IEEE Trans. Mob. Comp. 14 (6), 1162-1175.

Huang, M., Manton, J. H., 2009. Coordination and consensus of networked agents with noisy measurements: stochastic algorithms and asymptotic behavior. SIAM
Jnl on Cont. and Optim. 48 (1), 134-161.

Hui, P., Crowcroft, J., Yoneki, E., 2011. Bubble rap: Social-based forwarding in delay-tolerant networks. IEEE Trans. Mob. Comp. 10 (11), 1576-1589.

Jamieson, K. G., Nowak, R., 2011. Active ranking using pairwise comparisons. In: Proc. Neur. Inf. Proc. Syst. pp. 2240-2248.

Kantarci, B., Mouftah, H. T., August 2014. Trustworthy sensing for public safety in cloud-centric internet of things. IEEE Internet of Things Jnl 1 (4), 360-368.

Khabbaz, M. J., Assi, C. M., Fawaz, W. F., 2012. Disruption-tolerant networking: A comprehensive survey on recent developments and persisting challenges. IEEE Com. Surv. \& Tut. 14 (2), 607-640.

Lee, M.-H., Choi, Y.-H., 2008. Fault detection of wireless sensor networks. Comp. Com. 31 (14), 3469-3475.

Li, W., Bassi, F., Dardari, D., Kieffer, M., Pasolini, G., 2016a. Defective sensor identification for WSNs involving generic local outlier detection tests. IEEE Trans. on Sign. and Inf. Proc. over Netw. 2 (1), 29-48.

Li, W., Bassi, F., Galluccio, L., Kieffer, M., 2017. Peerassisted individual assessment in a multi-agent system. hal-01349638.

Li, W., Galluccio, L., Bassi, F., Kieffer, M., 2016b. Distributed faulty node detection in delay tolerant networks: Design and analysis. hal-01327472.

Luce, R. D., 1959. Individual choice behaviour: A theoretical analysis. Wiley, New-York.

Luo, P., Xiong, H., Lü, K., Shi, Z., 2007. Distributed classification in peer-to-peer networks. In: Proc. Int. Conf. on Knowl. Discov. and Data Mining. ACM, pp. 968-976.

Mahapatro, A., Khilar, P. M., 2013. Fault diagnosis in wireless sensor networks: A survey. IEEE Com. Surv. \& Tut. 15 (4), 2000-2026.

Negahban, S., Oh, S., Shah, D., 2012. Iterative ranking from pair-wise comparisons. In: Proc. Neur. Inf. Proc. Syst. Lake Tahoe, NE, pp. 2474-2482.

Orlinski, M., 2016. Encounter traces for the one simulator. Downloaded from http://www.shigs.co.uk/index.php?page =traces.

Ren, J., Zhang, Y., Zhang, K., Shen, X., July 2015. SACRM: Social Aware Crowdsourcing with Reputation Management in mobile sensing. Comp. Commun. 65.

Scott, J., Gass, R., Crowcroft, J., Hui, P., Diot, C., Chaintreau, A., May 2009. CRAWDAD dataset cambridge/haggle (v. 2009-05-29). Downloaded from http://crawdad.org/cambridge/haggle/20090529.

Shah, N. B., Balakrishnan, S., Wainwright, M. J., 2016. A permutation-based model for crowd labeling: Optimal estimation and robustness. arXiv preprint arXiv:1606.09632.

Thurstone, L., 1927. A law of comparative judgement. Psychological Review 34, 273-286.

Wauthier, F. L., Jordan, M. I., Jojic, N., 2013. Efficient ranking from pairwise comparisons. ICML (3) 28, 109 117.

Yick, J., Mukherjee, B., Ghosal, D., 2008. Wireless sen- 
sor network survey. Comp. Netw. 52 (12), 2292-2330.

Yu, Z., Van der Schaar, M., 2012. Reputation-based incentive protocols in crowdsourcing applications. In: Proc. IEEE INFOCOM. Orlando, FL, pp. 2140-2148. Zhang, Y., Meratnia, N., Havinga, P., 2010. Outlier detection techniques for wireless sensor networks: A survey. IEEE Com. Surv. \& Tut. 12 (2), 159-170.

Zhu, H., Fu, L., Xue, G., Zhu, Y., Li, M., Ni, L., March 2010. Recognizing exponential inter-contact time in vanets. In: Proc. IEEE INFOCOM. San Diego, CA, pp. $1-5$.

\section{Proofs}

\subsection{Proof of Proposition 1}

Consider a reference Agent $i$ with $c_{\mathrm{t}, i}=\tau<M$. In Case I, from (16), one has $\pi_{\theta}^{1,0}(t, \tau, \beta)+\pi_{\theta}^{1,1}(t, \tau, \beta)=1$. As a consequence, when Agent $i$ meets an other agent, $c_{\mathrm{t}, i}$ increases. Hence, $c_{\mathrm{t}, i}$ will reach $M$ after $M$ meetings and as $t \rightarrow \infty$, no node will be in a state $(\theta, \tau, \beta)$ with $\tau<M$. Hence, necessarily $\bar{X}_{\theta}^{\tau, \beta}=0$, for all $\tau<M$ and $\beta \leqslant \tau$.

The evaluation of $\bar{X}_{\theta}^{M, \beta}$ is similar to that considered in the proof of Proposition 3 in (Li et al., 2016b): the dynamics (3) can be simplified as $\boldsymbol{\Psi}_{\theta} \boldsymbol{X}_{\theta}^{M}(t)=\frac{d}{d t} \boldsymbol{X}_{\theta}^{M}(t)$, where $\boldsymbol{X}_{\theta}^{M}(t)=\left(\bar{X}_{\theta}^{M, 1}(t), \cdots, \bar{X}_{\theta}^{M, M}(t)\right)^{\mathrm{T}}$ and

$\boldsymbol{\Psi}_{\theta}=\left(\begin{array}{cccc}-a_{\theta}(0) & b_{\theta}(1) & & \\ a_{\theta}(0) & -a_{\theta}(1)-b_{\theta}(1) & b_{\theta}(2) & \\ & \ddots & \ddots & \ddots \\ & & a_{\theta}(M-1)-b_{\theta}(M)\end{array}\right)$,

with $a_{\theta}(\beta)=\pi_{\theta}^{0,1}(M, \beta)$ and $b_{\theta}(\beta)=\pi_{\theta}^{0,-1}(M, \beta)$. At equilibrium, one has $\frac{d}{d t} \boldsymbol{X}_{\theta}^{M}(t)=\mathbf{0}$. Thus, $\overline{\boldsymbol{X}}_{\theta}^{M}$ satisfies

$$
\boldsymbol{\Psi}_{\theta} \overline{\boldsymbol{X}}_{\theta}^{M}=\mathbf{0}
$$

In $(36)$, summing Lines 1 to $\beta+1$, for $\beta=0, \ldots, M-1$, one obtains $b_{\theta}(\beta+1) \bar{X}_{\theta}^{M, \beta+1}=a_{\theta}(\beta) \bar{X}_{\theta}^{M, \beta}$, hence

$$
\begin{aligned}
& \bar{X}_{\theta}^{M, \beta}=\bar{X}_{\theta}^{M, 0} \prod_{j=0}^{\beta-1} \frac{a_{0}(j)}{b_{0}(j+1)}=\bar{X}_{\theta}^{M, 0} \prod_{j=0}^{\beta-1} \frac{\pi_{\theta}^{0,1}(M, j)}{\pi_{\theta}^{0,-1}(M, j+1)} \\
& =\bar{X}_{\theta}^{M, 0} \prod_{j=0}^{\beta-1} \frac{(M-j) s_{\theta}}{(j+1)\left(1-s_{\theta}\right)}=\bar{X}_{\theta}^{M, 0}\left(\begin{array}{c}
M \\
\beta
\end{array}\right)\left(\frac{s_{\theta}}{1-s_{\theta}}\right)^{\beta} .
\end{aligned}
$$

Since $\sum_{\beta=0}^{M} \bar{X}_{\theta}^{M, \beta}=1$, one obtains for all $\theta \in \Theta$ and $\beta \in\{0, \ldots, M\} \bar{X}_{\theta}^{M, \beta}=\left(\begin{array}{c}M \\ \beta\end{array}\right)\left(s_{\theta}\right)^{\beta}\left(1-s_{\theta}\right)^{M-\beta}$.

\subsection{Proof of Proposition 2}

Consider an arbitrary $\theta \in \Theta$ and some $\varepsilon>0$ such that

$$
\varepsilon<\min _{1 \leqslant \theta<K} \frac{s_{\theta}-s_{\theta+1}}{2}
$$

Let $\Phi_{1}^{\theta}, \Phi_{2}^{\theta}, \ldots$ be an infinite sequence of i.i.d. binary random variables with $\mathbb{P}\left\{\Phi_{m}^{\theta}=1\right\}=s_{\theta}$. For any $\varrho \in$ 
$[0,1]$ such that $\varrho M \in \mathbb{N}^{+}$, one has

$$
\mathbb{P}\left\{\frac{\sum_{m=1}^{M} \Phi_{m}^{\theta}}{M}=\varrho\right\}=\left(\begin{array}{c}
M \\
\varrho M
\end{array}\right)\left(s_{\theta}\right)^{\varrho M}\left(1-s_{\theta}\right)^{M(1-\varrho)}
$$

According to the weak law of large numbers (Cover and Thomas, 2012), there exists $M^{\prime}$, such that for all $M$ > $M^{\prime}$, one has

$$
\mathbb{P}\left\{\left|\frac{\sum_{m=1}^{M} \Phi_{m}^{\theta}}{M}-s_{\theta}\right|<\varepsilon\right\}>1-\varepsilon
$$

From (39) and (40), one also has

$$
\begin{gathered}
\sum_{\beta: \beta / M \in\left(s_{\theta}-\varepsilon, s_{\theta}+\varepsilon\right)}\left(\begin{array}{c}
M \\
\beta
\end{array}\right)\left(s_{\theta}\right)^{\beta}\left(1-s_{\theta}\right)^{M-\beta} \\
=\mathbb{P}\left\{\frac{\sum_{m=1}^{M} \Phi_{m}^{\theta}}{M} \in\left(s_{\theta}-\varepsilon, s_{\theta}+\varepsilon\right)\right\}>1-\varepsilon .
\end{gathered}
$$

If $\nu_{\theta-1}$ and $\nu_{\theta}$ are chosen such that

$$
\nu_{\theta-1}>s_{\theta}+\varepsilon \text { and } s_{\theta}-\varepsilon>\nu_{\theta}
$$

then $\forall \theta \in \Theta$,

$$
\begin{aligned}
\bar{p}^{\theta \theta}=\sum_{\beta: \beta / M \in\left(\nu_{\theta}, \nu_{\theta-1}\right)}\left(\begin{array}{c}
M \\
\beta
\end{array}\right)\left(s_{\theta}\right)^{\beta}\left(1-s_{\theta}\right)^{M-\beta} \\
>\sum_{\beta: \beta / M \in\left(s_{\theta}-\varepsilon, s_{\theta}+\varepsilon\right)}\left(\begin{array}{c}
M \\
\beta
\end{array}\right)\left(s_{\theta}\right)^{\beta}\left(1-s_{\theta}\right)^{M-\beta}>1-\varepsilon .
\end{aligned}
$$

The constraints (42) have to be satisfied for $\theta=1 \ldots K$. They may be reformulated as

$$
s_{\theta+1}+\varepsilon<\nu_{\theta}<s_{\theta}-\varepsilon
$$

for $\theta=1 \ldots K-1$. Such values of $\nu_{\theta}$ may be found, since one imposes the constraints (38) on $\varepsilon$.

One concludes that $\forall \theta \in \Theta$ and $\forall \varepsilon>0$ satisfying (38), there exists a choice of the values of $\nu_{\theta}$ such that (43) is satisfied and there exists $M^{\prime}$, such that for all $M>M^{\prime}$, $\bar{p}^{\theta \theta}>1-\varepsilon$. The value of $\varepsilon$ can be chosen arbitrarily close to zero, which will require $M$ going to infinity, so, provided that $s_{\theta+1}<\nu_{\theta}<s_{\theta}, \forall \theta \in \Theta$, one has $\lim _{M \rightarrow \infty} \bar{p}^{\theta \theta}=1$, if $\nu_{\theta}<s_{\theta}<\nu_{\theta-1}, \forall \theta \in \Theta$.

\subsection{Proof of Proposition 4}

According to Proposition 3, one has $\bar{X}_{\theta}^{\tau, \beta}=0$, for all $\beta \leqslant \tau<M$. Using derivations similar to (37), one gets

$$
\begin{aligned}
\bar{X}_{\theta}^{M, \beta} & =\bar{X}_{\theta}^{M, 0} \prod_{j=0}^{\beta-1} \frac{\pi_{\theta}^{0,1}(M, j)}{\pi_{\theta}^{0,-1}(M, j+1)} \\
& =\bar{X}_{\theta}^{M, 0} \prod_{j=0}^{\beta-1} \frac{(M-j) \sum_{k \in \Theta} p_{k} \bar{p}^{k 1} q(\theta, k)}{(j+1) \sum_{k \in \Theta} p_{k} \bar{p}^{k 1}(1-q(\theta, k))} \\
& =\bar{X}_{\theta}^{M, 0}\left(\begin{array}{c}
M \\
\beta
\end{array}\right)\left(\frac{h_{\theta}}{1-h_{\theta}}\right)^{\beta}
\end{aligned}
$$

with $h_{\theta}$ defined in (20). Since $\sum_{\beta=0}^{M} \bar{X}_{\theta}^{M, \beta}=1$, one obtains for all $\theta \in \Theta$ and $\beta \in\{0, \ldots, M\}$

$$
\bar{X}_{\theta}^{M, \beta}=\left(\begin{array}{c}
M \\
\beta
\end{array}\right) h_{\theta}^{\beta}\left(1-h_{\theta}\right)^{M-\beta} .
$$

Introducing (45) in (12), one obtains (23) with $F_{\theta}$ defined in (21). Thus one needs to solve (23) to determine $\bar{p}^{\theta 1}$ for all $\theta \in \Theta$. The $\bar{p}^{\theta 1} \mathrm{~s}$ are then used to deduce $\bar{X}_{\theta}^{M, \beta}$ applying (45).

\subsection{Proof of Proposition 5}

Brouwer's fixed-point theorem (Granas and Dugundji, 2013) can be used to show the existence of a solution of (23). For that purpose, one has to show that for any $\mathbf{p}^{1}(0)=\left(p^{11}(0) \ldots p^{K 1}(0)\right) \in \mathcal{P}_{0}=\{\boldsymbol{x} \in$ $[0,1]^{K}$ and $\left.\boldsymbol{x} \neq \mathbf{0}\right\}$, the discrete-time system

$$
\mathbf{p}^{1}(n+1)=\mathbf{F}\left(\mathbf{p}^{1}(n)\right),
$$

with $\mathbf{p}^{1}(n)=\left(p^{11}(n) \ldots p^{K 1}(n)\right) \forall n \in \mathbb{N}^{*}$, converges to a equilibrium point $\overline{\mathbf{p}}^{1}$.

Each $F_{\theta}$ with $\theta \in \Theta$ is a continuous function. One needs to verify whether the value $\mathbf{p}^{1}(0)$ belongs to some compact set.

Lemma 8 One always has $\sum_{\theta \in \Theta} p^{\theta 1}(t)>0, \forall t \in \mathbb{R}^{+}$.

Proof The proof is similar to that of Lemma 9 and 10 in ( $\mathrm{Li}$ et al., 2016b).

From Lemma 8, one obtains that

$$
\mathbf{p}^{1}(0) \in \mathcal{P}_{0}=\left\{\boldsymbol{x} \in[0,1]^{K} \text { such that } \boldsymbol{x} \neq \mathbf{0}\right\} .
$$

$\mathcal{P}_{0}$ is not compact. One then has to find a compact $\mathcal{P}_{n}$ such that $\mathbf{F}$ maps $\mathcal{P}_{n}$ to $\mathcal{P}_{n}$, in order to apply Brouwer's fixed-point. One starts showing some properties of some basic functions involved in $\mathbf{F}$. 
Lemma 9 If $\boldsymbol{x}=\left(x_{1} \ldots x_{K}\right) \in \mathcal{P}_{0}$, then $h_{\theta}(\boldsymbol{x})$ is bounded as follows

$$
0<h_{\theta, \min } \leqslant h_{\theta}(\boldsymbol{x}) \leqslant h_{\theta, \max }
$$

where

$$
\left\{\begin{array}{l}
h_{\theta, \max }=\max \{q(\theta, 1) \ldots q(\theta, K)\} \\
h_{\theta, \min }=\min \{q(\theta, 1) \ldots q(\theta, K)\}
\end{array}\right.
$$

Proof Using Assumption A6), one has $h_{\theta, \min }=$ $\min \{q(\theta, 1) \ldots q(\theta, K)\}>0$. Moreover, one has

$$
\begin{aligned}
h_{\theta, \max }-h_{\theta}(\boldsymbol{x}) & =h_{\theta, \max }-\frac{\sum_{k \in \Theta} p_{k} q(\theta, k) x_{k}}{\sum_{k \in \Theta} p_{k} x_{k}} \\
& =\frac{\sum_{k \in \Theta} p_{k}\left(h_{\theta, \max }-q(\theta, k)\right) x_{k}}{\sum_{k \in \Theta} p_{k} x_{k}} .
\end{aligned}
$$

Since $h_{\theta, \max } \geqslant q(\theta, k), \forall k \in \Theta$, one gets $h_{\theta, \max }-$ $h_{\theta}(\boldsymbol{x}) \geqslant 0$. In a similar way,

$$
h_{\theta, \min }-h_{\theta}(\boldsymbol{x})=\frac{\sum_{k \in \Theta} p_{k}\left(h_{\theta, \min }-q(\theta, k)\right) x_{k}}{\sum_{k \in \Theta} p_{k} x_{k}} \leqslant 0 .
$$

Then (48) is proved.

Lemma 10 If $0<\nu_{1}<1$, the function

$$
g(z)=\sum_{\beta=\left\lceil M \nu_{1}\right\rceil}^{M}\left(\begin{array}{c}
M \\
\beta
\end{array}\right) z^{\beta}(1-z)^{M-\beta}
$$

is increasing for all $z \in[0,1]$.

Proof In (Li et al., 2016b), Lemma 13, one has shown that $1-g(z)$ is decreasing for $z \in[0,1]$, thus $g(z)$ is increasing.

From Lemma 9 and Lemma 10, one obtains that for any $\theta \in \Theta$ and $\boldsymbol{x} \in \mathcal{P}_{0}$

$$
0<g\left(h_{\theta, \min }\right) \leqslant F_{\theta}(\boldsymbol{x}) \leqslant g\left(h_{\theta, \max }\right) .
$$

Define $p_{\max }^{\theta 1}(n)$ and $p_{\text {min }}^{\theta 1}(n)$ as upper and lower bounds of $p^{\theta 1}(n)$, i.e., $p_{\min }^{\theta 1}(n) \leqslant p^{\theta 1}(n) \leqslant p_{\max }^{\theta 1}(n)$. When $n=0, p_{\min }^{\theta 1}(0)=0$ and $p_{\max }^{\theta 0}(0)=1$. From (51), one gets

$$
\left\{\begin{array}{l}
p_{\min }^{\theta 1}(1)=g\left(h_{\theta, \min }\right)>0 \\
p_{\max }^{\theta 1}(1)=g\left(h_{\theta, \max }\right)
\end{array}\right.
$$

Define

$$
\mathcal{P}_{1}=\left[p_{\min }^{11}(1), p_{\max }^{11}(1)\right] \times \ldots \times\left[p_{\min }^{K 1}(1), p_{\max }^{K 1}(1)\right],
$$

then $\mathbf{p}^{1}(1)=\mathbf{F}\left(\mathbf{p}^{1}(0)\right) \in \mathcal{P}_{1}$. Notice that $\mathbf{0} \notin \mathcal{P}_{1}$ and $\mathcal{P}_{1}$ is a compact set since $p_{\min }^{\theta 1}(1)>0$.
Consider then an arbitrary integer $n \in \mathbb{N}^{*}$. Assume that $\mathbf{p}^{1}(n-1) \in \mathcal{P}_{1}$, one needs to see whether $\mathbf{p}^{1}(n) \in \mathcal{P}_{1}$ is satisfied. Since $\mathcal{P}_{1} \subseteq \mathcal{P}_{0}$, one still has

$$
h_{\theta, \min } \leqslant h_{\theta}\left(\mathbf{p}^{1}(n-1)\right) \leqslant h_{\theta, \max },
$$

which leads to

$$
g\left(h_{\theta, \min }\right) \leqslant p^{\theta 1}(n)=F_{\theta}\left(\mathbf{p}^{1}(n-1)\right) \leqslant g\left(h_{\theta, \max }\right) .
$$

Therefore $\mathbf{F}$ maps $\mathcal{P}_{1}$ to $\mathcal{P}_{1}$. Besides, $\mathcal{P}_{1}$ is compact. Hence one can apply Brouwer's fixed-point theorem to prove Proposition 5.

\subsection{Proof of Proposition 6}

First, one investigates the upper bound of $\bar{p}^{\theta 1}$ for $\theta \in$ $\Theta \backslash\{1\}$ and $M$ sufficient large. As for (41), for any $\varepsilon>0$, there exists $m_{1}$, such that for all $M>m_{1}$

$$
\sum_{\beta=\left\lfloor\left(s_{\theta}-\varepsilon\right) M\right\rfloor+1}^{\left\lceil\left(s_{\theta}+\varepsilon\right) M\right\rceil-1}\left(\begin{array}{c}
M \\
\beta
\end{array}\right)\left(h_{\theta, \max }\right)^{\beta}\left(1-h_{\theta, \max }\right)^{M-\beta}>1-\varepsilon,
$$

which leads to

$$
\begin{aligned}
& \sum_{\beta=\left\lfloor\left(h_{\theta, \max }+\varepsilon\right) M\right\rfloor+1}^{M}\left(\begin{array}{c}
M \\
\beta
\end{array}\right)\left(h_{\theta, \max }\right)^{\beta}\left(1-h_{\theta, \max }\right)^{M-\beta} \\
& <1-\sum_{\beta=\left\lfloor\left(s_{\theta}-\varepsilon\right) M\right\rfloor+1}^{\left\lceil\left(s_{\theta}+\varepsilon\right) M\right\rceil-1}\left(\begin{array}{c}
M \\
\beta
\end{array}\right)\left(h_{\theta, \max }\right)^{\beta}\left(1-h_{\theta, \max }\right)^{M-\beta} \\
& <\varepsilon .
\end{aligned}
$$

If $\nu_{1}>h_{\theta, \max }+\varepsilon$, then from (53) and (54), one gets

$$
\begin{aligned}
& \bar{p}^{\theta 1}<\sum_{\beta=\left\lceil\nu_{1} M\right\rceil}^{M}\left(\begin{array}{c}
M \\
\beta
\end{array}\right)\left(h_{\theta, \max }\right)^{\beta}\left(1-h_{\theta, \max }\right)^{M-\beta} \\
& <\sum_{\beta=\left\lfloor\left(h_{\theta, \max }+\varepsilon\right) M\right\rfloor+1}^{M}\left(\begin{array}{c}
M \\
\beta
\end{array}\right)\left(h_{\theta, \max }\right)^{\beta}\left(1-h_{\theta, \max }\right)^{M-\beta}<\varepsilon .
\end{aligned}
$$

Moreover, if

$$
\nu_{1}>\max _{\theta \in \Theta \backslash\{1\}}\left(h_{\theta, \max }+\varepsilon\right)=\max _{\theta \in \Theta \backslash\{1\}, k \in \Theta}(q(\theta, k)+\varepsilon),
$$

then $\bar{p}^{\theta 1}<\varepsilon, \forall \theta \in \Theta \backslash\{1\}$.

Second, one derives the lower bound of $\bar{p}^{11}$. Denote $\zeta=$ 
$\min _{\theta \in \Theta \backslash\{1\}} q(1, \theta)$, one has

$$
\begin{aligned}
h_{1}\left(\overline{\mathbf{p}}^{1}\right) & =\frac{p_{1} q(1,1) \bar{p}^{11}+\sum_{k \in \Theta \backslash\{1\}} p_{k} q(1, k) \bar{p}^{k 1}}{\sum_{k \in \Theta} p_{k} \bar{p}^{k 1}} \\
& \geqslant \frac{p_{1} q(1,1) \bar{p}^{11}+\zeta \sum_{k \in \Theta \backslash\{1\}} p_{k} \bar{p}^{k 1}}{\sum_{k \in \Theta} p_{k} \bar{p}^{k 1}} \\
& >\frac{p_{1} q(1,1) p_{\min }^{11}(1)+\zeta \varepsilon \sum_{k \in \Theta \backslash\{1\}} p_{k}}{p_{1} p_{\min }^{11}(1)+\varepsilon \sum_{k \in \Theta \backslash\{1\}} p_{k}}=\chi .
\end{aligned}
$$

According to Lemma 10, one has $\bar{p}^{11}>g(\chi)$. If $\nu_{1}<$ $\chi-\varepsilon, \bar{p}^{11}$ can be further bounded as

$$
\begin{aligned}
\bar{p}^{11}> & g(\chi)=\sum_{\beta=\left\lceil\nu_{1} M\right\rceil}^{M}\left(\begin{array}{c}
M \\
\beta
\end{array}\right) \chi^{\beta}(1-\chi)^{M-\beta} \\
> & \sum_{\beta=\lfloor(\chi-\varepsilon) M\rfloor+1}^{M}\left(\begin{array}{c}
M \\
\beta
\end{array}\right) \chi^{\beta}(1-\chi)^{M-\beta} \\
\geqslant & \sum_{\beta=\lfloor(\chi-\varepsilon) M\rfloor+1}^{\lceil(\chi+\varepsilon) M\rceil-1}\left(\begin{array}{c}
M \\
\beta
\end{array}\right) \chi^{\beta}(1-\chi)^{M-\beta} \stackrel{(a)}{>} 1-\varepsilon .
\end{aligned}
$$

Again, similar to (41), there exists $m_{2}>m_{1}$ such that for all $M>m_{2},(a)$ is true.

Notice that $\lim _{\varepsilon \rightarrow 0} \chi=q(1,1)$, one obtains that if

$$
\max _{\theta \in \Theta \backslash\{1\}, k \in \Theta} q(\theta, k)<\nu_{1}<q(1,1),
$$

then $\lim _{M \rightarrow \infty} \bar{p}^{11}=1$. Using similar derivations, if $q(\theta+$ $1,1)<\nu_{\theta}<q(\theta, 1)$, for all $\theta=1 \ldots K-1$, one has $\lim _{M \rightarrow \infty} \bar{p}^{\theta \theta}=1, \forall \theta \in \Theta \backslash\{1\}$.

\subsection{Proof of Proposition 7}

One starts with Case I. To lighten the notations, let $d_{\theta}=$ $\left\lceil\nu_{\theta} M\right\rceil$. From $(26)$, one needs to find $\mathbf{d}^{*}=\left(d_{1}^{*} \ldots d_{K-1}^{*}\right)$ such that $d_{1}^{*}>\ldots>d_{K-1}^{*}$ and

$$
\begin{array}{r}
U(\mathbf{d})=\sum_{\theta \in \Theta} \bar{p}^{\theta \theta}=\sum_{d=d_{1}}^{M}\left(\begin{array}{c}
M \\
\beta
\end{array}\right)\left(s_{1}\right)^{\beta}\left(1-s_{1}\right)^{M-\beta} \\
+\sum_{\theta=2}^{K} \sum_{d=d_{\theta}}^{d_{\theta}-1}\left(\begin{array}{c}
M \\
\beta
\end{array}\right)\left(s_{\theta}\right)^{\beta}\left(1-s_{\theta}\right)^{M-\beta}
\end{array}
$$

takes maximum value as $\mathbf{d}=\mathbf{d}^{*}$.

Consider an arbitrary $\theta \in \Theta$, one evaluates

$$
\begin{aligned}
& \delta_{\theta}=U\left(d_{1} \ldots, d_{\theta}+1, \ldots d_{K-1}\right)-U\left(d_{1} \ldots, d_{\theta}, \ldots d_{K-1}\right) \\
& =\left(\begin{array}{c}
M \\
d_{\theta}
\end{array}\right)\left(\left(s_{\theta+1}\right)^{d_{\theta}}\left(1-s_{\theta+1}\right)^{M-d_{\theta}}-\left(s_{\theta}\right)^{d_{\theta}}\left(1-s_{\theta}\right)^{M-d_{\theta}}\right) .
\end{aligned}
$$

If $\delta_{\theta}>0$, then

$$
\left(s_{\theta+1}\right)^{d_{\theta}}\left(1-s_{\theta+1}\right)^{M-d_{\theta}}>\left(s_{\theta}\right)^{d_{\theta}}\left(1-s_{\theta}\right)^{M-d_{\theta}},
$$

leading to

$$
d_{\theta}<M \frac{\log \left(\frac{1-s_{\theta}}{1-s_{\theta+1}}\right)}{\log \left(\frac{s_{\theta+1}}{s_{\theta}} \frac{1-s_{\theta}}{1-s_{\theta+1}}\right)}=\widetilde{d}_{\theta},
$$

notice that $\log \left(\frac{s_{\theta+1}}{s_{\theta}} \frac{1-s_{\theta}}{1-s_{\theta+1}}\right)<0$ as $s_{\theta+1}<s_{\theta}$.

In contrast, if $\delta_{\theta}<0$, then $d_{\theta}>\widetilde{d}_{\theta}$. As a conclusion, for any $d_{1}>\ldots d_{\theta} \ldots>d_{K-1}$, one has

$$
U\left(d_{1} \ldots, d_{\theta}, \ldots d_{K}\right) \leqslant U\left(d_{1} \ldots, d_{\theta}^{*}, \ldots d_{K}\right),
$$

$\forall \theta=1 \ldots(K-1)$, where

$$
d_{\theta}^{*}=\left\lceil\widetilde{d}_{\theta}\right\rceil=\left\lceil M \frac{\log \left(\frac{1-s_{\theta}}{1-s_{\theta+1}}\right)}{\log \left(\frac{s_{\theta+1}}{s_{\theta}} \frac{1-s_{\theta}}{1-s_{\theta+1}}\right)}\right\rceil .
$$

Then one has

$$
\begin{aligned}
& U\left(d_{1} \ldots d_{K-1}\right) \leqslant U\left(d_{1}^{*}, d_{2} \ldots d_{K-1}\right) \\
& \leqslant U\left(d_{1}^{*}, d_{2}^{*}, d_{3} \ldots d_{K-1}\right) \leqslant \ldots \leqslant U\left(d_{1}^{*} \ldots d_{K}^{*}\right) .
\end{aligned}
$$

Replacing $s_{\theta}$ with $q(\theta, 1)$ and using the same derivations, one obtains the $\nu_{\theta}$ s which maximize $\sum_{\theta \in \Theta} \widetilde{p}^{\theta \theta}$. 